\title{
Connecting Theory with Experiment to Understand the Sintering Processes of Ag Nanoparticles
}

Edison Z. da Silva, ${ }^{* \dagger \dagger}$ Giovani M. Faccin, ${ }^{\ddagger}$ Thales R. Machado, "Marcelo Assis, Nadia G. Macedo, Santiago Maya-Johnson, "Júlio C. Sczancoski, Juan Andrés, ${ }^{\S}$ Elson Longo, " and Miguel A. San-Miguell

†Institute of Physics "Gleb Wataghin", UNICAMP, CP 6165, 13083-9859, Campinas - SP, Brazil

$\ddagger$ Faculdade de Ciências Exatas e Tecnológicas, Universidade Federal da Grande Dourados Unidade II, CP 533, 79804-970, Dourados - MS, Brazil

\CDMF, LIEC, Federal University of São Carlos (UFSCar), P.O. Box 676, 13565-905 São Carlos, SP, Brazil

$\S$ Department of Analytical and Physical Chemistry, University Jaume I (UJI), Castelló 12071, Spain

|| Instituto de Química, UNICAMP, CP 6165, 13083-970, Campinas - SP, Brazil

E-mail: zacarias@ifi.unicamp.br

\begin{abstract}
A complementary combination of long-time atomistic molecular dynamics (MD) simulations and real-time transmission electron microscopy (TEM) images has been utilized to unraveling, for the first time and at atomic resolution, the nature of the sintering process of $\mathrm{Ag}$ nanoparticles (NPs) induced on the surface of $\alpha-\mathrm{Ag}_{2} \mathrm{WO}_{4}$ crystal
\end{abstract}


under the exposure of an electron beam (EB) of the TEM. Temporal evolution of both calculated and experimental results highlight the role of the lattice plane matching and the stacking faults along the disorder to order transitions of the oriented attachment process. This phenomenon is understood as an example of surface plasmon resonances (SPR), in which the electron beam has two effects: first, it provokes the formation of the Ag NPs that due to the electron irradiation become SPR electric dipoles and second, these Ag NPs undergo sintering processes that are controlled by dipole-dipole interactions forming larger clusters. The predictive power of the simulation model is verified experimentally, paving the way toward quantitative predictions of the involved events along the Ag NPs sintering process. These findings reveal the atomic-scale dynamics, which helps to advance our general understanding and prove further support and reliability of our conclusions.

The electron beam (EB) irradiation provided by transmission electron microscopy (TEM) is a well established technique for characterization into the nano and angstrom scale, in which high energy electrons transmit through the specimen. The analysis of energy exchanges between the electrons and the sample adds further information on the chemical composition and electronic structure of the samples based on electron-solid interactions, which is of significance both for fundamental research and the potential technological applications. ${ }^{1-4}$ There are many evidences of the formation and growth of Ag nanoparticles (NPs) within the area irradiated by the EB on different Ag-based micro/nanostructures. ${ }^{1,5-11}$ These syntheses, that employ the EB of the TEM, are particularly valuable since the microscopes allow one to peer into the structural evolution at nanoscale of the Ag NPs as they are being synthesized. Five years ago, our group started a research line based on the observation of the formation of $\mathrm{Ag}$ NPs on different Ag-containing compounds ( $\alpha-\mathrm{Ag}_{2} \mathrm{WO}_{4},{ }^{12-15} \beta-\mathrm{Ag}_{2} \mathrm{WO}_{4},{ }^{16,17}$ $\beta-\mathrm{Ag}_{2} \mathrm{MoO}_{4},{ }^{18} \mathrm{Ag}_{2} \mathrm{CrO}_{4},{ }^{19} \beta-\mathrm{AgVO}_{3},{ }^{20} \mathrm{Ag}_{3} \mathrm{PO}_{4}{ }^{21}$ ) induced by the irradiation of an electron EB from a TEM in vacuum. These works opened the door for a series of both experimental and theoretical studies to reach interesting possibilities of producing Ag NPs on these materials because the synergism between the support semiconductor and Ag NPs can enhance 
their stability and the corresponding performance in promising technological applications as photoluminescence and bactericide materials. ${ }^{22-24}$ In this context, the very recent review of Zhang et al. ${ }^{25}$ highlights the progress in the synthesis of Ag NPs and the formulation of sintered Ag paste with enhanced mechanical, electrical and thermal conductivity properties.

Sintering process governs the initial stage of the junction of the grain boundaries in which two initially isolated particles bond together to form larger particles. This is an activated process and it plays a prominent role in controlling several properties of the resulting particle, such as the particle size, and the particle size distribution. Sintering of metal NPs has been investigated in the past decade both experimentally ${ }^{12,13,26-31}$ and also computationally. ${ }^{29,32-36}$ These processes involve at least three stages: the approach between NPs, the oriented attachment (OA) involving pre-aligned crystallographic orientations between them or imperfect oriented attachment (IOA) when touching occurs with different surface planes and angles, and the subsequent coalescence or aggregation process to form a single NP. The understanding of nanoparticle sintering is therefore essential for obtaining nanoparticles or nanocrystalline materials with desired physical and chemical properties. Detailed knowledge on these stages along the sintering process and the interaction between active NPs is thus of considerable importance, but still poses a significant challenge. ${ }^{37,38}$ Molecular dynamics (MD) simulation represents a powerful tool to provide deep insights on the molecular mechanisms that drive and control the interactions between NPs. By solving the equation of motion for a large system of particles, the real behavior of materials can be simulated under a specified temperature. The trajectory and physical movement of NPs in the system can also be determined and analyzed. Recently, MD simulations have been successfully used to investigate the sintering process of different metals $(\mathrm{Al}, \mathrm{Cu} \text { and } \mathrm{Ni})^{36,39,40}$ and metal oxides $\left(\mathrm{TiO}_{2}{ }^{41}\right.$ and $\left.\mathrm{CuO}^{42}\right)$.

Surface plasmon resonances (SPR) effects appear in metal NPs and they are associated to the frequencies of collective oscillations of the free electrons that fall in the optical range 
of the electromagnetic spectrum. ${ }^{43,44}$ Plasmonic metal NPs can concentrate incident optical fields in nanometer-sized volumes. This leads high local fields not only to enhance optical responses but also to induce chemical transformation, driven by plasmon-induced hot carriers (optical spectroscopies). ${ }^{45}$ Actually, plasmons were first revealed as energy loss features in the spectra of electrons rejected from metal surfaces. ${ }^{46}$ Since then, EBs have become an important tool to yield information on plasmons. ${ }^{47-49}$ Light could be used as the energy source in the synthesis of $\mathrm{Ag} \mathrm{NPs}^{50,51}$ and $\mathrm{Au}$ NPs. ${ }^{52,53}$ Previous studies of metal NPs have shown that, similarly to the case of laser irradiation, EB irradiation also produces electromagnetic wave radiation (EWR) that acts on the NPs producing SPR nanodipoles. In this process, Ag NPs are wavelength (or frequency) filters capable of absorbing EWR in specific frequencies, which excite SPR on the Ag NPs. ${ }^{54-56}$ Therefore nearby Ag NPs in bonding states attract each other and this is the driving effect to start the sintering process.

\section{Kinetics of NP sintering}

The kinetics of NP sintering is governed by processes on two quite different length scales. The longer length scale involves the diffusive transport of heat, while the intrinsic growth rate, in contrast, is governed by the nanoscopic dynamics associated with the cooperative reorganization at the NPs interface. In this study, direct atomic resolution was conducted to explore the fundamental understanding of the sintering mechanism of Ag NPs formed on the $\alpha-\mathrm{Ag}_{2} \mathrm{WO}_{4}$ material under EB irradiation. The novelty of this work relates to the fact that we collect essential information at atomic level, from real in situ TEM images and MD simulations to study the interactions between the Ag NPs that under the EWR developed into SPR resonances (nanoelectrical dipoles), and then the nature of the sintering mechanism is disclosed. These are necessary for studying structural evolution, changing growth regimes, and providing insights into the sintering progress. The study started with the exploration of the trajectories of Ag NPs by observing the coalescence events of the different Ag NPs. 
For most of the time, Ag NPs keep moving randomly; but sometimes they are found along the viewing zone axis and despite occasional off-axis fluctuations, it is possible to identify their orientations by lattice fringes. Understanding the analogies between the experimental and simulated scenarios implies new strategies for rational comprehension of the sintering process. Based on this knowledge, the following four questions will be answered: How the formation of $\mathrm{Ag} \mathrm{NPs}$, in the range from 4 to $8 \mathrm{~nm}$, induced by EB irradiation on $\alpha-\mathrm{Ag}_{2} \mathrm{WO}_{4}$ crystals in vacuum, takes place? What physical parameters determine the rate of Ag NPs aggregation? What is the role of the stacking faults along the disorder to order transitions of the sintering process? What is the physical origin responsible for the sintering process of $\mathrm{Ag}$ NPs provoked by the interaction between matter and EB?

\section{Real-time sintering of Ag NPs}

A schematic representation of the studied phenomena by a joint use of experimental and theoretical methods is depicted in Fig. 1. The EB of TEM provokes the segregation of the metal $\mathrm{Ag}^{0}$ atoms from the bulk to the surface of the $\alpha-\mathrm{Ag}_{2} \mathrm{WO}_{4}$ crystal, with concomitant formation and growth of $\mathrm{Ag}$ nanoparticles labeled as "mother $\mathrm{Ag}^{0}$ particles" on top of the surface. As reported in previous studies with $\alpha-\mathrm{Ag}_{2} \mathrm{WO}_{4}$, as soon as the EB hits the semiconductor sample, the Ag nucleation process begins (Step 1). ${ }^{12,13,22,23}$ This nucleation process is due to the action of the electrons from the beam, which are transferred to the material from one cluster to another through the lattice network. The Ag nucleation is a consequence of the reduction of the $\mathrm{Ag}^{+}$in different extents of the $\left[\operatorname{Ag} O_{x}\right],(x=2,4,6,7)$ clusters into $\mathrm{Ag}^{0}$, which promotes a transformation from an ordered structure of $\alpha-\mathrm{Ag}_{2} \mathrm{WO}_{4}$ to a disordered one. The growth of $\mathrm{Ag} \mathrm{NPs}$ on $\alpha-\mathrm{Ag}_{2} \mathrm{WO}_{4}$ is a consequence of structural order/disorder effects generated on the semiconductor material when the EB passes through it. ${ }^{14,26}$ This phenomena is similar to the grain boundary migration-dominated pathway proposed by Li et al. to explain the crystal nucleation and growth initiated from an amorphous 
state of Bi metal under electron beam inside an aberration-corrected transmission electron microscope ${ }^{57}$. Once the Ag NPs nucleation and growth happens on Step 1, the incoming electrons promote new phenomena on the studied system. ${ }^{14}$ Thus, not only the formation of $\mathrm{Ag}$ NPs on the surface of $\alpha-\mathrm{Ag}_{2} \mathrm{WO}_{4}$ at initial instants can be observed, but also, the continued exposition to the EB can lead to a fragmentation process of the as-formed $\mathrm{Ag}$ NPs and subsequent appearance of free Ag NPs in vacuum (Step 2). ${ }^{14}$ This phenomenon is a consequence of the increasing exposition of the large initial Ag NPs to the EB, which makes it unstable, experiencing several structural changes, until the occurrence of an explosive reaction, with the ejection outwards of a large number of Ag NPs. This is a well-known process that results from the transfer of thermal energy and electric charge, ${ }^{58}$ and takes place at metallic samples which can melt and collapse into units as the irradiating current density increases. The new formed Ag NPs can be expelled and "fly" in the carbon grid for some nanometers of distance, as observed. These Ag NPs become electric dipoles and they are the building blocks to start the coalescence process (Step 3), as shown in Fig. 1. This phenomenon is a consequence of the increasing exposition of the large initial Ag NPs to the EB, which makes it unstable, experiencing several structural changes, until the occurrence of the an explosive reaction, with the ejection outwards of a large number of Ag NPs. ${ }^{1}$ The sizes of the Ag NPs are in the range from 4 to $8 \mathrm{~nm}$ and their aggregation or coalescence process occur through motion of the Ag NPs driven by the electron forces ending in a mass transfer process that produces larger and well defined Ag NPs, with more stable configurations. ${ }^{14,59}$ For a detailed description of the experimental procedure employed, refer to section S1 of the Supporting Information (SI). A video of the coalescence step is available in the SI section (Video S1).

The experiments show the temporal evolution of the free flying Ag NPs and their interactions forming other larger Ag NPs. These processes can be understood from Mie theory, as an example of SPR effects in which neutral dipole interactions are used to explain them. The interaction among the Ag NPs comprises three different types: orientation and induction, 


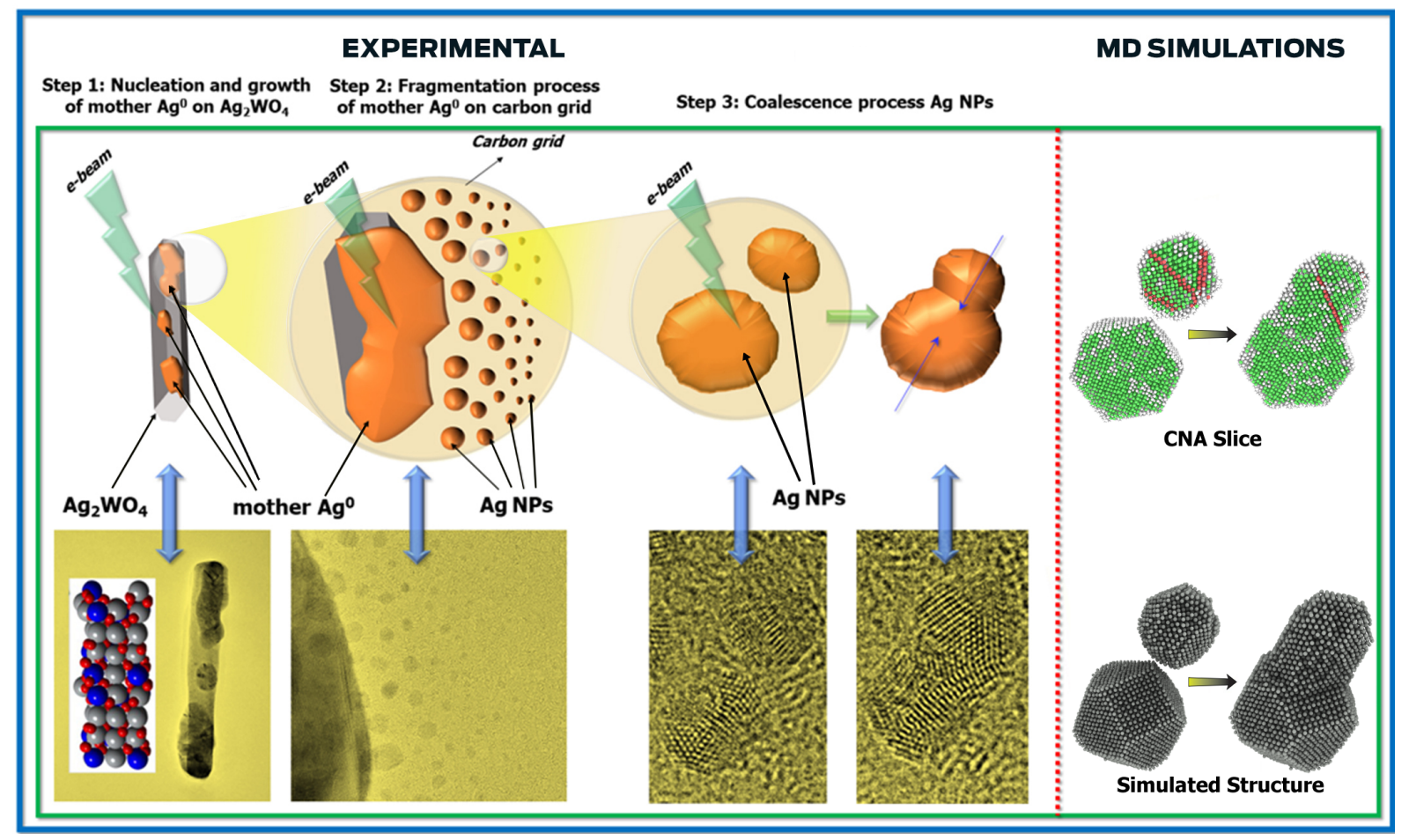

Figure 1: A schematic representation of experimental and theoretical results for the present study. The sintering process starts with the nucleation and growth of mother $\mathrm{Ag}^{0}$ particles on $\alpha-\mathrm{Ag}_{2} \mathrm{WO}_{4}$ (step 1). The exposure of the EB from TEM induces the reduction of the $\mathrm{Ag}$ cations which migrate from the $\alpha-\mathrm{Ag}_{2} \mathrm{WO}_{4}$ bulk crystal structure to form mother $\mathrm{Ag}^{0}$ on the $\mathrm{Ag}_{2} \mathrm{WO}_{4}$ surface. When the EB is focused on these mother $\mathrm{Ag}^{0}$, a fragmentation process takes place in which smaller Ag NPs become free on the carbon grid (Step 2). These free Ag NPs are involved in the aggregation or coalescence processes (Step 3). To further understand the last step, MD simulations were performed. 

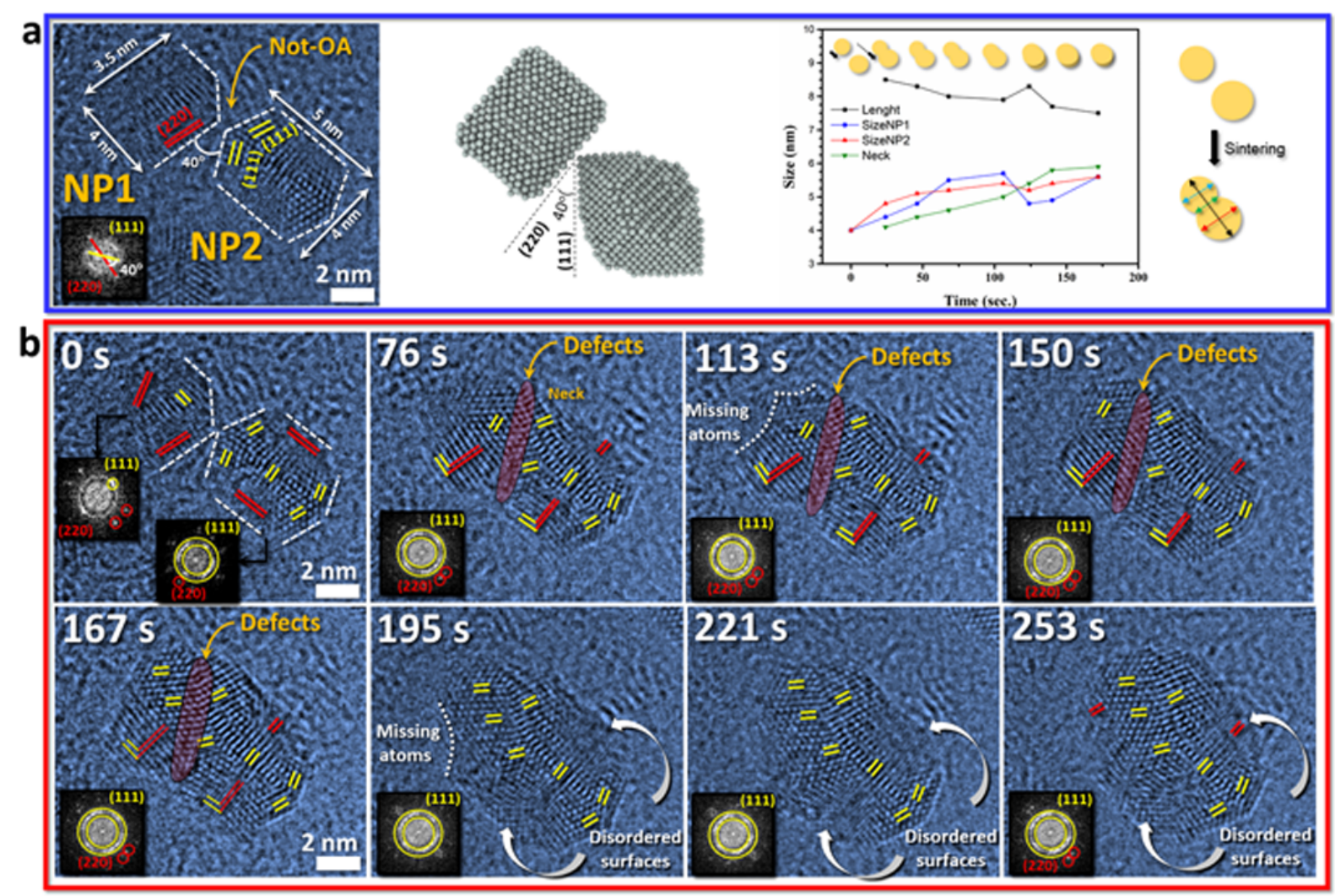

Figure 2: Sintering events of the Ag NPs. (a) HR-TEM images of two Ag NPs, NP1 and NP2 (left). Both Ag NPs are multifaceted and the main exposed planes, (220) and (111), are displayed in this image. The fast Fourier transformation (FFT), at the inset, shows the crystallographic planes near the contact region of these Ag NPs, evidencing an initially disorder driven event by the estimation of angle between the (220) and (111) planes (red and yellow lines, respectively). These Ag NPs were simulated, and the results are presented at the center of the figure. Estimations of the particles and necks sizes with distinct exposure times to the EB (right). (b) Sequence of HR-TEM images evidencing the coalescence process of the Ag NPs under the EB exposure. The FFTs (insets) obtained from these Ag NPs show the main crystallographic planes, (220) and (111), illustrated as red and yellow lines, respectively. 
that can be explained by the classical theory, and dispersion that can only be rationalized by the quantum mechanics. The orientation interaction results from the correlation between the rotation motion of the permanent moments of the Ag NPs, while the induction interaction is due to the polarization of the Ag NP by the permanent moment of another Ag NP. The dispersion interaction arises from the correlation of the motion of electrons in neighboring clusters. ${ }^{60,61}$ Since the Ag NPs under the EB become SPR nanodipoles with Ag NPs of sizes ranging from 4 to $8 \mathrm{~nm}$, these nearby Ag NPs interact via dipole-dipole interaction and this leads to the formation of dimers that contact to start a coalescence process, forming new $\mathrm{Ag}$ NPs, as was observed in the present experiments. Therefore, we believe that these events are clear evidence of the mechanism described here. The Ag NPs touch with different surface planes, therefore the coalescence process discussed in this work does not involve crystalline oriented attachment, for reasons that will be discussed. ${ }^{62}$ The mechanism underlying this unusual in situ Ag NPs formation requires careful examination based on a physical basis ground. To this end, and as valuable complement to such experimental observations, a comprehensive study has been carried out using MD simulation methods which provides insights into the structural changes and energetic properties of the Ag NPs, to unveil the details of the coalescence process in order to complement the experimental findings.

Fig. 2 shows the experimental results obtained for two well-defined Wulff shaped Ag NPs, NP1 and NP2, with different exposed facets. In each surface, distinct planes can be indexed, mainly related to (220) and (111), in accordance with JCPDS database (PDF - $04-0783)$ for face-centered cubic (fcc) metallic $\mathrm{Ag}^{0}$ structure. Initially, at time $0 \mathrm{~s}$ (Fig. 2a, left), just after the fragmentation process of mother Ag NP, the newly formed Ag NPs are separated by $0.4 \mathrm{~nm}$ at the closest distance of the point between the (220) surface of NP1 and the corner formed by (111) facets of NP2. The angle between these crystallographic planes was measured by identifying their Fourier reflections (inset), giving a value of $40^{\circ}$. Hence, the distinct planes which are exposed at these surfaces as well as their misalignment lead to an initially disorder driven process. The dimensions of these Ag NPs are quite similar: $3.5 \mathrm{x}$ 
$4.0 \mathrm{~nm}(\mathrm{NP} 1)$ and $4.0 \times 5.0 \mathrm{~nm}$ (NP2). Fig. 2a (center) illustrates the Ag NPs used in the simulations; it shows their contact angles, where a good agreement between the experimental and theoretical simulations is observed. The exposure of the Ag NPs to the EB leads to the coalescence process and longer exposure times also gives energy to the formation and growth of the neck for the sinterised Ag NPs. Fig. 2a (right) shows the evolution of the Ag NPs and neck sizes as function of exposure time.

In order to clarify these events at the atomic level, we monitored at distinct exposure times the effect on the Ag NPs by a time-resolved electron microscopy from $0 \mathrm{~s}$ to $253 \mathrm{~s}$, and Fig. 2b shows the obtained results. In these images, we can sense different phenomena with high spatial resolution occurring during the welding processes: rotation of Ag NPs in order to minimize the crystallographic mismatch, growth of the neck between the two Ag NPs, atomic diffusion mainly from facets with exposed (220) planes, ordered/disordered transition of these facets, and formation/healing of the disordered interface. To accomplish the understanding of these events, we emphasize the principal crystallographic planes which reorganize during coalescence process both in real images and by fast Fourier transform (FFT) as well as the observed defects. The FFT of the initial NPs (0 s) and the posteriorly formed dimer consists of the typical ones from faceted NPs. Hence, a partial halo (yellow circles in the insets) with specific reflections is observed, which departs mainly from (111) plane at distinct facets as well as from (200) planes (not shown). Other reflections outside this region (red circles in the insets) are observed mainly from (220) planes. The reflections in FTT match well with the crystallographic planes demonstrated in the real images. Taking into account all these observations, it can be assumed that the coalescence process of the $\mathrm{Ag}$ NPs in the present work is accompanied by neck-growing formed between the Ag NPs due to diffusion of surface atoms and not a simple rigid-body collision.

Initially, at time $0 \mathrm{~s}$, an important mismatch is observed between the (220) and (111) planes from both Ag NPs, which greatly decreases after $76 \mathrm{~s}$ probably due to a rotation of Ag NPs. However, the lattice alignment between the Ag NPs remains imperfect, and 
line dislocations (brown region labeled as defects) are created as an effect of crystal-crystal incoherent bonding, leading to a defect-mediated coalescence of the Ag NPs. Hence, the Ag NPs reorient to a limited extent, and the resulting configuration allows defects to form, as a result, misoriented attachment. These defects formed during bonding persist from many seconds until $167 \mathrm{~s}$, where a sudden ordering is observed. During this healing process of the interface, it is also observed the neck growth and diffusion of mobile Ag atoms (labeled as missing atoms) mainly departing from facets with (220) exposed planes. At the final stage of coalescence process from $167 \mathrm{~s}$ to $253 \mathrm{~s}$, three main effects can be summarized: (i) continuous growth of the neck and decrease of facets shared; (ii) increase of crystalline coherency between (111) exposed planes of both Ag NPs leading to a close-to-oriented bonding; (iii) disordering of facets, probably due to a high mobility of the Ag atoms in these facets (labeled as disordered surfaces). Moreover, the two FFT spots corresponding to these facets alternate from defined to various diffuse spots depending on irradiation time between $167 \mathrm{~s}$ and 253 s. These findings could be associated to a melted state of these less stable surfaces as an effect of the interaction with the high-energy EB. Hence, these atoms could be the major contributors for increase ordering, neck size, and crystalline coherency of the final sintered Ag NP.

\section{Connection to theory: coalescence simulations}

Our aim here is to understand at a fundamental level the nanoscopic processes underlying the experimental data discussed above. The sintering process of two Ag NPs will involve many competing effects, such as surface diffusion due to preferential melting of high energy surface atoms as well as internal competition between the formed disordered/ordered interfaces, preventing healing and thus perfect welding. The present MD simulations provide a careful study of how two Ag NPs are capable to form a larger one, similar to those observed in the experiments (see Figs. S1 to S5 in SI for details). 
The main idea that explains the reason for the interaction between Ag NPs has been discussed previously ${ }^{34}$ and can be explained with the help of Mie theory. As shown by Koh et al. ${ }^{54}$ metal Ag NPs in the presence of the EB become SPR objects. This occurs because Ag NPs are wavelength (or frequency) filters for this EWR and the resonance results in SPR neutral objects. ${ }^{54-56,63}$ These SPRs behave as nano electric dipoles. Therefore, nearby NPs in the SPR state can form bonding and antibonding states due to the fact that they behave as nanodipoles. Nearby NPs in bonding states attract each other and this is the driving effect to promote sintering.

Detailed MD simulations have been carried out to understand the coalescence process. Previous study ${ }^{33}$ has highlighted the key role of the temperature along the different scenarios. When two Ag NPs are approaching and the touching interface is defect free and display similar facets, an oriented attachment process ${ }^{64}$ takes place, while when there is a plane mismatch at the interface between both Ag NPs, defects are formed along IOA. ${ }^{65,66}$

The present work stresses the importance of long time simulations to capture different events that occur at different times during the coalescence and sintering. Snapshots of the simulation, with a total time interval of $\Delta t=290 \mathrm{~ns}$, are shown in Fig. 3. Figure 3a shows snapshots of the coalescence process with two views; for each time label two images are shown, on top the atomic structure and at bottom the common neighbor analysis (CNA) cut that gives information of the internal structure of the Ag NP. In order to help understanding the overall evolution, some important distances are measured for the structures of Fig. 3a and are presented in Fig 3b, namely, the length of the formed Ag NP (black line), the interface neck (green line) and also the sizes of the two particles radii (blue line for NP1 and red line for NP2). The evolution shows a clear decrease in the Ag NP size and a clear increase of the neck length, as it should be for the coalescence process; these results show reasonably well the same trends of the evolution seen in the experimental images. Figure 3a shows snapshots starting with the contact of the $\operatorname{Ag} \operatorname{NPs}(t=00 \mathrm{~ns})$, as in the experiment, with a sharp edge formed by (111) surfaces of NP2 touching the (220) facet of NP1 at 40 (SI Fig. S5). 
A rotation of NP1 occurs in the early stages of attachment and evolves as the simulation progresses; this can be seen at $\mathrm{t}=40 \mathrm{~ns}$ (see SI, Fig. S6 and Fig. S7). Therefore, orientation occurs in order to accommodate the contacting planes but the touching surfaces are very different; thus this process is a disordered driven attachment event, for the short time span of $\Delta t=5 \mathrm{~ns}$. Despite the rotation, the new Ag NP ends up with a disordered interface. While the rotation is understood from the Ag NP outer structure as in the experiments, the CNA cut images help us understand the type of interface produced (see SI for the $\Delta t=5 \mathrm{~ns}$, Fig. S8): it is a disordered interface and some defected regions are also formed, initially in NP2. Upon contact the experiments show a rotation, also observed in the early stages of the MD simulations. The merging occurs accompanied by the formation of a disordered interface, which lowers surface energy and is maintained during the initial moments of the simulation (see Fig. S8). It is important to note that this process is similar to IOA mechanism observed by Penn and Banfield ${ }^{65}$ and Li et al. ${ }^{66}$ in which NPs did not fully correct misalignment during attachment and the resulting interface consisted of grain boundary dislocations. It is well known that if the interface started ordered, the total energy of the new structure would be further lowered since defects are more energetic. At $t=60 \mathrm{~ns}$ an unexpected ordering event occurred. The consequence of this event is a sudden lowering of the energy shown in Fig. 3c, typical of phase transitions. In fact, we just obtained a disorder to order transition. This striking event is shown in Fig. 3a for $t=60 \mathrm{~ns}$ and a detailed evolution of this effect in time steps of $\Delta t=10 \mathrm{~ns}$ is shown in Fig S9. Insets in the energy plot in Fig. 3c and the detailed evolution is timesteps of $\Delta t=0.1 \mathrm{~ns}$ in Fig. 3d show details of the phase transition, in which the amorphous interface is pushed towards NP1. This conjunction of events, initially disordered driven, forming a disordered interface that later evolve to an ordered interface due to the disorder to order transition, can be viewed as a two stage ordering event. This effect could only be observed due to the extended simulation time considered in the present study. This is important because in the experiments, the time interval between images are longer and this concerted behavior could be missed, with the ordered interface that resulted being 
considered as simple OA, that due to the initial conditions, was not expected. Another effect of the ordering transition is that the occurrence of the new order allows the hcp stacking fault to evolve and permeate the whole extension of the Ag NP producing at the surface ends, disordered and mobile regions that can provide mobile atoms for surface diffusion as well as prevent the better faceting that Wulff structures search for (SI Fig. S9).

a
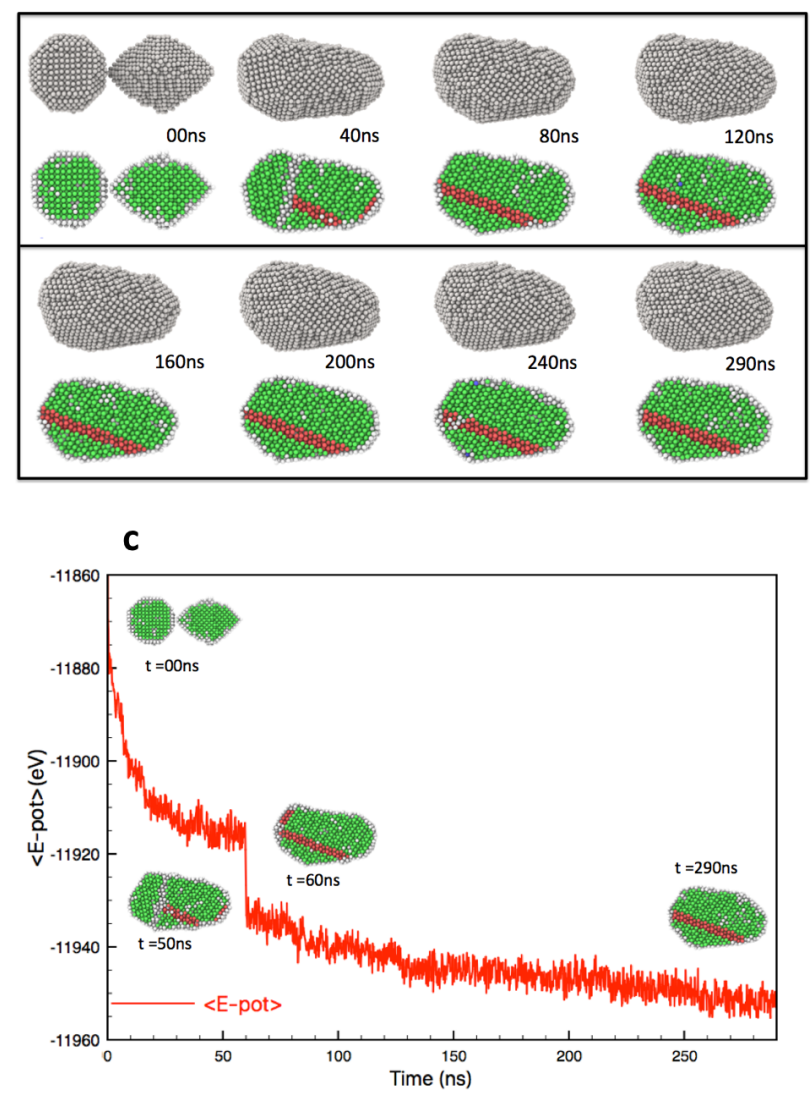

b

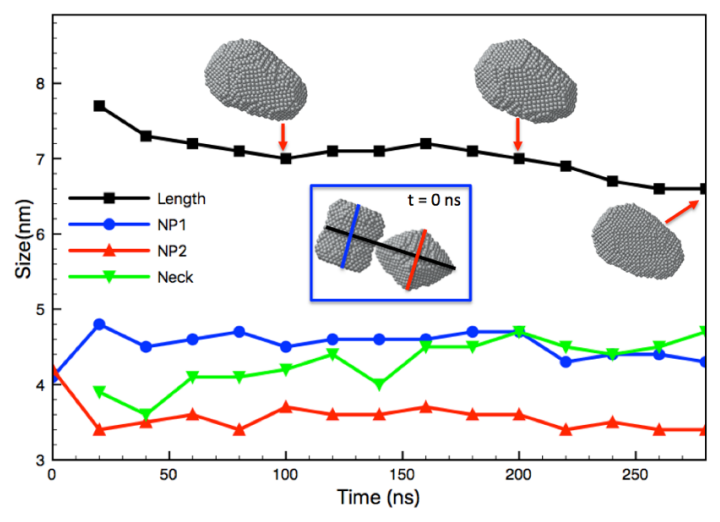

d

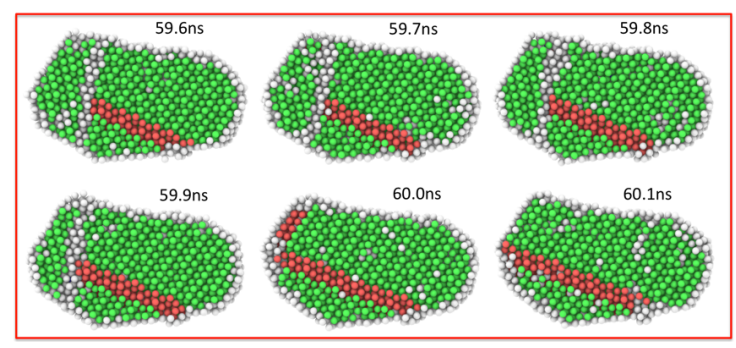

Figure 3: MD simulation of two attached Ag NPs: a) Structures and CNA slice images at different times. b) Evolution of characteristic lengths associated to the process; length of NP (black line), lateral length of NP1 (blue line) and NP2 (red line) and also the size of the interface neck (green line). Inset, NP1 and NP2 prior to contact. c) Average potential energy, CNA insets show initial and final structures as well as the two images associated with the disorder to order transition. d) Detailed time evolution of the process for the disorder to order transition. 


\section{Conclusions}

Exposure of Ag-containing materials to the EB unveiled new and interesting phenomena. Understanding the dynamics of NPs formation and growth mechanisms is of great interest as the mode of assembling can influence the type of periodic lattice which is formed, and hence the properties of the resulting nanocrystals. Watching the movements and dynamics of NPs and the subsequent assembling processes at high vacuum under the EB of a TEM provide helpful new insights. A complementary combination of atomistic MD simulations and in situ TEM images has been utilized to increase our limited understanding of the sintering of $\mathrm{Ag} \mathrm{NPs}$, in the range from 4 to $8 \mathrm{~nm}$, provoked by the EB irradiation on $\alpha-\mathrm{Ag}_{2} \mathrm{WO}_{4}$ crystals in vacuum.

The conclusions of the present work can be summarized as follows: i) the EB beam that is used to image the sample, carries an electromagnetic field that produces SPR in the Ag NPs, forming neutral nanoelectric dipoles that are responsible for the corresponding coalescence processes. Then, our study yields a unique playground for the observation of the fundamental properties of SPR in confined electromagnetic fields, explained here as a dimer interaction between Ag NPs, ii) MD simulations unveiled that the coalescence process of the Ag NPs start with a disordered driven pathway or IOA mechanism followed by a structural disorder to order transition, via an OA mechanism. It also showed how healing and/or evolution of stacking faults contribute to the formation of the Ag NP, iii) we believe that the results presented herein shed new light on the various factors that dictate SPR-mediated coalescence processes in NPs and inspire the understanding of plasmonic processes that can be triggered and/or controlled by the EBs, iv) The mechanisms discussed herein enable the synthesis of Ag NPs and, possibly, other structures in which the NPs are the building blocks that interact with the EB of the TEM, v) The general methodology and insights gained from our depth study strengthen our understanding of sintering control on EB treatment, since structural and energetic information are encoded on the evolution of Ag NPs. 


\section{Methods}

Methods, including statements of data availability and any associated accession codes and references, are available at (fill DOI address when available).

Received: ; Accepted: ;Published online: (fill dates when available).

\section{References}

1. Gonzalez-Martinez, I. G.; Bachmatiuk, A.; Bezugly, V.; Kunstmann, J.; Gemming, T.; Liu, Z.; Cuniberti, G.; Rummeli, M. H. Electron-beam induced synthesis of nanostructures: a review. Nanoscale 2016, 8, 11340-11362.

2. Liu, Y.; Lin, X.-M.; Sun, Y.; Rajh, T. In situ Visualization of Self-Assembly of Charged Gold Nanoparticles. Journal of the American Chemical Society 2013, 135, 3764-3767, PMID: 23432699.

3. Huang, C.-W.; Chen, J.-Y.; Chiu, C.-H.; Hsin, C.-L.; Tseng, T.-Y.; Wu, W.-W. Observing the evolution of graphene layers at high current density. Nano Research 2016, 9, 3663-3670.

4. Liu, X.; Xu, T.; Wu, X.; Zhang, Z.; Yu, J.; Qiu, H.; Hong, J.-H.; Jin, C.-H.; Li, J.-X.; Wang, X.-R. et al. Topdown fabrication of sub-nanometre semiconducting nanoribbons derived from molybdenum disulfide sheets. Nat. Commun. 2013, 4, 1776.

5. Umalas, M.; Vlassov, S.; Polyakov, B.; Dorogin, L. M.; Saar, R.; Kink, I.; Lhmus, R.; Lhmus, A.; Romanov, A. E. Electron beam induced growth of silver nanowhiskers. Journal of Crystal Growth 2015, 410, $63-68$.

6. Makita, Y.; Ikai, O.; Hosokawa, J.; Ooi, K.; Okuyama, S.; Sumida, N. Synthesis of Long Silver Nanowires by Electron Beam Irradiation on Ag-exchanged Material. Journal of Ion Exchange 2003, 14, 409-412. 
7. Edmondson, M. J.; Zhou, W.; Sieber, S. A.; Jones, I. P.; Gameson, I.; Anderson, P. A.; Edwards, P. P. Electron-Beam Induced Growth of Bare Silver Nanowires from Zeolite Crystallites. Advanced Materials 2001, 13, 1608-1611.

8. Yuan, Z.-Y.; Zhou, W.; Parvulescu, V.; Su, B.-L. Electron beam irradiation effect on nanostructured molecular sieve catalysts. Journal of Electron Spectroscopy and Related Phenomena 2003, 129, 189 -194.

9. Li, K.; Zhang, F.-S. A novel approach for preparing silver nanoparticles under electron beam irradiation. Journal of Nanoparticle Research 2010, 12, 1423-1428.

10. Yoji, M.; Osamu, I.; Akira, O.; Kenta, O. Preparation of Long Silver Nanowires from Silver Matrix by Electron Beam Irradiation. Chemistry Letters 2002, 31, 928-929.

11. Ma, C.; Chen, X.; Tan, X.; Hu, P.; Li, Q.; Cao, Y.; Liang, X. In situ fabrication of silver-based nanostructures using electron beam. CrystEngComm 2018, 20, 2227-2232.

12. Longo, E.; Cavalcante, L.; Volanti, D.; et. al., Direct in situ observation of the electrondriven synthesis of Ag filaments on $\alpha-\mathrm{Ag}_{2} \mathrm{WO}_{4}$ crystals. Scientific Reports 2013, 3, 1676.

13. Andrés, J.; Gracia, L.; P., G.-N.; Longo, V.; Avansi, W.; Volanti, D.; Ferrer, M.; Lemos, P.; La Porta, F.; Hernandes, A. et al. Structural and electronic analysis of the atomic scale nucleation of $\mathrm{Ag}$ on $\alpha-\mathrm{Ag}_{2} \mathrm{WO}_{4}$ induced by electron irradiation. Scientific Reports 2014, 4, 5391.

14. Longo, E.; Avansi, W.; Bettini, J.; Andrés, J.; Gracia, L. In situ Transmission Electron Microscopy observation of Ag nanocrystal evolution by surfactant free electron-driven synthesis. Scientific Reports 2016, 6, 21498.

15. San-Miguel, M. A.; da Silva, E. Z.; Zanetti, S. M.; Cilense, M.; Fabbro, M. T.; Gracia, L.; Andrés, J.; Longo, E. In situ growth of $\mathrm{Ag}$ nanoparticles on $\alpha-\mathrm{Ag}_{2} \mathrm{WO}_{4}$ under electron irradiation: probing the physical principles. Nanotechnology 2016, 27, 225703. 
16. Roca, R. A.; Gouveia, A. F.; Lemos, P. S.; Gracia, L.; Andrés, J.; Longo, E. Formation of $\mathrm{Ag}$ Nanoparticles on $\beta-\mathrm{A} g_{2} \mathrm{WO}_{4}$ through Electron Beam Irradiation: A Synergetic Computational and Experimental Study. Inorganic Chemistry 2016, 55, 8661-8671.

17. Roca, R. A.; Lemos, P. S.; Andrés, J.; Longo, E. Formation of Ag nanoparticles on metastable $\beta-\mathrm{Ag}_{2} \mathrm{WO}_{4}$ microcrystals induced by electron irradiation. Chemical Physics Letters 2016, 644, $68-72$.

18. Fabbro, M. T.; Saliby, C.; Rios, L. R.; Porta, F. A. L.; Gracia, L.; Li, M. S.; Andrés, J.; Santos, L. P. S.; Longo, E. Identifying and rationalizing the morphological, structural, and optical properties of $\alpha-\mathrm{Ag}_{2} \mathrm{MoO}_{4}$ microcrystals, and the formation process of $\mathrm{Ag}$ nanoparticles on their surfaces: combining experimental data and first-principles calculations. Science and Technology of Advanced Materials 2015, 16, 065002.

19. Fabbro, M. T.; Gracia, L.; Silva, G. S.; Santos, L. P.; Andrés, J.; Cordoncillo, E.; Longo, E. Understanding the formation and growth of Ag nanoparticles on silver chromate induced by electron irradiation in electron microscope: A combined experimental and theoretical study. Journal of Solid State Chemistry 2016, 239, 220 - 227.

20. de Oliveira, R. C.; Assis, M.; Teixeira, M. M.; da Silva, M. D. P.; Li, M. S.; Andrés, J.; Gracia, L.; Longo, E. An Experimental and Computational Study of $\beta-\mathrm{Ag}_{2} \mathrm{VO}_{3}$ : Optical Properties and Formation of Ag Nanoparticles. The Journal of Physical Chemistry C 2016, 120, 12254-12264.

21. Botelho, G.; Sczancoski, J. C.; Andrés, J.; Gracia, L.; Longo, E. Experimental and Theoretical Study on the Structure, Optical Properties, and Growth of Metallic Silver Nanostructures in $\mathrm{Ag}_{3} \mathrm{PO}_{4}$. The Journal of Physical Chemistry C 2015, 119, 6293-6306.

22. Longo, V. M.; De Foggi, C. C.; Ferrer, M. M.; Gouveia, A. F.; André, R. S.; Avansi, W.; Vergani, C. E.; Machado, A. L.; Andrés, J.; Cavalcante, L. S. et al. Potentiated Electron 
Transference in $\alpha-\mathrm{Ag}_{2} \mathrm{WO}_{4}$ Microcrystals with $\mathrm{Ag}$ Nanofilaments as Microbial Agent. The Journal of Physical Chemistry A 2014, 118, 5769-5778.

23. Longo, E.; Volanti, D. P.; Longo, V. M.; Gracia, L.; Nogueira, I. C.; Almeida, M. A. P.; Pinheiro, A. N.; Ferrer, M. M.; Cavalcante, L. S.; Andrés, J. Toward an Understanding of the Growth of $\mathrm{Ag}$ Filaments on $\alpha-\mathrm{A} g_{2} \mathrm{WO}_{4}$ and Their Photoluminescent Properties: A Combined Experimental and Theoretical Study. The Journal of Physical Chemistry C 2014, 118, 1229-1239.

24. Liu, D.; Huang, W.; Li, L.; Liu, L.; Sun, X.; Liu, B.; Yang, B.; Guo, C. Experimental and theoretical investigation on photocatalytic activities of $1 \mathrm{D} \mathrm{Ag} / \mathrm{Ag}_{2} \mathrm{WO}_{4}$ nanostructures. Nanotechnology 2017, 28, 385702.

25. Zhang, P.; Jiang, X.; Yuan, P.; Yan, H.; Yang, D. Silver nanopaste: Synthesis, reinforcements and application. International Journal of Heat and Mass Transfer 2018, 127, $1048-1069$.

26. Pereira, W. d. S.; Andrés, J.; Gracia, L.; San-Miguel, M. A.; da Silva, E. Z.; Longo, E.; Longo, V. M. Elucidating the real-time Ag nanoparticle growth on $\alpha$-A $g_{2} \mathrm{WO}_{4}$ during electron beam irradiation: experimental evidence and theoretical insights. Phys. Chem. Chem. Phys. 2015, 17, 5352-5359.

27. Mansourian, A.; Paknejad, S. A.; Zayats, A. V.; Mannan, S. H. Stereoscopic NanoscalePrecision Growth of Free-Standing Silver Nanorods by Electron Beam Irradiation. The Journal of Physical Chemistry C 2016, 120, 20310-20314.

28. Grouchko, M.; Roitman, P.; Zhu, X.; Popov, I.; Kamyshny, A.; Su, H.; Magdassi, S. Merging of metal nanoparticles driven by selective wettability of silver nanostructures. Nature Communications 2014, 5, 2994.

29. Grammatikopoulos, P.; Cassidy, C.; Singh, V.; Sowwan, M. a. Coalescence-induced crystallisation wave in Pd nanoparticles. Scientific Reports 2014, 4, 5779. 
30. José-Yacamán, M.; Gutierrez-Wing, C.; Miki, M.; Yang, D.-Q.; Piyakis, K. N.; Sacher, E. Surface Diffusion and Coalescence of Mobile Metal Nanoparticles. The Journal of Physical Chemistry B 2005, 109, 9703-9711, PMID: 16852169.

31. Lange, A.; Samanta, A.; Majidi, H.; Mahajan, S.; Ging, J.; Olson, T.; van Benthem, K.; Elhadj, S. Dislocation mediated alignment during metal nanoparticle coalescence. Acta Materialia 2016, 120, $364-378$.

32. Pereira, Z. S.; da Silva, E. Z. Cold Welding of Gold and Silver Nanowires: A Molecular Dynamics Study. The Journal of Physical Chemistry C 2011, 115, 22870-22876.

33. Faccin, G. M.; San-Miguel, M. A.; Andrés, J.; Longo, E.; da Silva, E. Z. Computational Modeling for the Ag Nanoparticle Coalescence Process: A Case of Surface Plasmon Resonance. The Journal of Physical Chemistry C 2017, 121, 7030-7036.

34. Andrés, J.; Gouveia, A. F.; Gracia, L.; Longo, E.; Manzeppi Faccin, G.; da Silva, E. Z.; Pereira, D. H.; San-Miguel, M. A. Formation of Ag nanoparticles under electron beam irradiation: Atomistic origins from first-principles calculations. International Journal of Quantum Chemistry 2018, 118, e25551.

35. Li, M.; Hou, Q.; Wang, J. A molecular dynamics study of coalescence of tungsten nanoparticles. Nuclear Instruments and Methods in Physics Research Section B: Beam Interactions with Materials and Atoms 2017, 410, 171 - 178.

36. Cheng, B.; Ngan, A. H. The crystal structures of sintered copper nanoparticles: A molecular dynamics study. International Journal of Plasticity 2013, 47, 65-79.

37. Gutiérrez-Wing, C.; Olmos-Asar, J.; Esparza, R.; Mariscal, M.; Yacamán, M. The role of ad-atoms in the coalescence of alkanethiol-passivated gold nanoparticles. Electrochimica Acta 2013, 101, $301-307$. 
38. Hansen, T. W.; DeLaRiva, A. T.; Challa, S. R.; Datye, A. K. Sintering of Catalytic Nanoparticles: Particle Migration or Ostwald Ripening? Accounts of Chemical Research 2013, 46, 1720-1730, PMID: 23634641.

39. Li, Y.; Kalia, R. K.; Nakano, A.; Vashishta, P. Size effect on the oxidation of aluminum nanoparticle: Multimillion-atom reactive molecular dynamics simulations. Journal of Applied Physics 2013, 114, 134312.

40. Xu, J.; Sakanoi, R.; Higuchi, Y.; Ozawa, N.; Sato, K.; Hashida, T.; Kubo, M. Molecular Dynamics Simulation of Ni Nanoparticles Sintering Process in Ni/YSZ MultiNanoparticle System. The Journal of Physical Chemistry C 2013, 117, 9663-9672.

41. Buesser, B.; Gröhn, A. J.; Pratsinis, S. E. Sintering Rate and Mechanism of $\mathrm{TiO}_{2}$ Nanoparticles by Molecular Dynamics. The Journal of Physical Chemistry C 2011, 115, 11030-11035, PMID: 23730400.

42. Zhao, H.; Gui, J.; Cao, J.; Zheng, C. Molecular Dynamics Simulation of the Microscopic Sintering Process of CuO Nanograins Inside an Oxygen Carrier Particle. The Journal of Physical Chemistry $C$ 2018, in press.

43. Stockman, M. I. Nanoplasmonics: past, present, and glimpse into future. Opt. Express 2011, 19, 22029-22106.

44. Halas, N. J.; Lal, S.; Chang, W.-S.; Link, S.; Nordlander, P. Plasmons in Strongly Coupled Metallic Nanostructures. Chemical Reviews 2011, 111, 3913-3961.

45. Szunerits, S.; Boukherroub, R. Introduction to Plasmonics: Advances and Applications; CRC Press: Boca Raton, FL 33487-2742, 2015.

46. Powell, C. J.; Swan, J. B. Origin of the Characteristic Electron Energy Losses in Aluminum. Phys. Rev. 1959, 115, 869-875. 
47. Nelayah, J.; Kociak, M.; Stephan, O.; Garcia de Abajo, F. J.; Tence, M.; Henrard, L.; Taverna, D.; Pastoriza-Santos, I.; Liz-Marzan, L. M.; Colliex, C. Mapping surface plasmons on a single metallic nanoparticle. Nature Physics 2007, 3, 348-353.

48. Liz-Marzán, L. M. Plasmonics. Electron Oscillations and Beyond. The Journal of Physical Chemistry Letters 2013, 4, 1197-1198.

49. Alvarez-Puebla, R.; Liz-Marzán, L. M.; Garca de Abajo, F. J. Light Concentration at the Nanometer Scale. The Journal of Physical Chemistry Letters 2010, 1, 2428-2434.

50. Xu, X.; Liu, G.; Azad, A. K. Visible light photocatalysis by in situ growth of plasmonic $\mathrm{Ag}$ nanoparticles upon $\mathrm{AgTaO}_{3}$. International Journal of Hydrogen Energy 2015, 40, $3672-3678$.

51. Lu, Y.; Shen, Q.; Yu, Q.; Zhang, F.; Li, G.; Zhang, W. Photoinduced in situ Growth of Ag Nanoparticles on $\mathrm{AgNbO}_{3}$. The Journal of Physical Chemistry C 2016, 120, 2871228716.

52. Brus, L. Plasmon-driven chemical synthesis: Growing gold nanoprisms with light. Nat. Mater. 2016, 15, 824-825.

53. Zhai, Y.; DuChene, J. S.; Wang, Y.-C.; Qiu, J.; Johnston-Peck, A. C.; You, B.; Guo, W.; DiCiaccio, B.; Qian, K.; Zhao, E. W. et al. Polyvinylpyrrolidone-induced anisotropic growth of gold nanoprisms in plasmon-driven synthesis. Nat. Mater. 2016, 15, 889-895.

54. Koh, A. L.; Bao, K.; Khan, I.; Smith, W. E.; Kothleitner, G.; Nordlander, P.; Maier, S. A.; McComb, D. W. Electron Energy-Loss Spectroscopy (EELS) of Surface Plasmons in Single Silver Nanoparticles and Dimers: Influence of Beam Damage and Mapping of Dark Modes. ACS Nano 2009, 3, 3015-3022.

55. Prodan, E.; Radloff, C.; Halas, N. J.; Nordlander, P. A Hybridization Model for the Plasmon Response of Complex Nanostructures. Science 2003, 302, 419-422. 
56. Prodan, E.; Nordlander, P. Plasmon hybridization in spherical nanoparticles. The Journal of Chemical Physics 2004, 120, 5444-5454.

57. Li, J.; Chen, J.; Wang, H.; Chen, N.; Wang, Z.; Guo, L.; Deepak, F. L. In Situ AtomicScale Study of Particle-Mediated Nucleation and Growth in Amorphous Bismuth to Nanocrystal Phase Transformation. Advanced Science 2018, 5, 1700992.

58. Pyrz, W. D.; Park, S.; Vogt, T.; Buttrey, D. J. Electron Beam-Induced Fragmentation and Dispersion of BiNi Nanoparticles. The Journal of Physical Chemistry C 2007, 111, $10824-10828$.

59. Assis, M. M.; Machado, T. R.; Ferrer, M. M.; Gouveia, A. F.; Cordoncillo, E.; Mendieta, R. O.; Beltrán-Mir, H.; Andrés, J.; Longo, E. Laser/electron irradiation on InP semiconductor: A promising pathway to in situ formation of In nanoparticles. Part. Part. Syst. Charact 2018, in press.

60. Hill, T. L. Statistical Mechanics; McGraw-Hill, 1956.

61. Sinanoglu, O. Modern Quantum Chemistry; Academic Press, 1965.

62. Aabdin, Z.; Lu, J.; Zhu, X.; Anand, U.; Loh, N. D.; Su, H.; Mirsaidov, U. Bonding Pathways of Gold Nanocrystals in Solution. Nano Letters 2014, 14, 6639-6643.

63. Nordlander, P.; Oubre, C.; Prodan, E.; Li, K.; Stockman, M. I. Plasmon Hybridization in Nanoparticle Dimers. Nano Letters 2004, 4, 899-903.

64. Wang, Y. Q.; Liang, W. S.; Geng, C. Y. Coalescence Behavior of Gold Nanoparticles. Nanoscale Research Letters 2009, 4, 684.

65. Penn, R. L.; Banfield, J. F. Imperfect Oriented Attachment: Dislocation Generation in Defect-Free Nanocrystals. Science 1998, 281, 969-971. 
66. Li, D.; Nielsen, M. H.; Lee, J. R. I.; Frandsen, C.; Banfield, J. F.; De Yoreo, J. J. Direction-Specific Interactions Control Crystal Growth by Oriented Attachment. Science 2012, 336, 1014-1018.

\section{Acknowledgements}

The authors thank the National Center for High Performance Computing in São Paulo (CENAPAD-SP) and the CCJDR-UNICAMP for providing the computational resources for this project. The following agencies contributed funds for this research: Coordenação de Aperfeiçoamento de pessoal de nível superior - Brasil (CAPES) - Finance code 001, PNPD Program, FINEP, FAPESP (2013/07296-2), CNPq (150205/2017-1), UFGD, Generalitat Valenciana for Prometeo II/2014/022, ACOMP/2015/1202, Ministerio de Economia y Competitividad, project CTQ2015-65207-P, and Universitat Jaume I project No. UJI-B2016-25. The authors also thank Enio Longo for design contributions.

\section{Author contributions}

The manuscript was done through the contributions of all authors. J. Andrés (J.A.), E. Longo (E.L.), M. San-Miguel (M.S.), and E. Z. da Silva (E.Z.S) conceived the project. E.L. conceived the idea of the experiment, while E.Z.S., M.S. and G. M. Faccin (G.M.F.) conceived the idea of molecular dynamics (MD) simulations. J.C. Sczancoski (J.C.S.) synthesized the initial $\mathrm{Ag}_{2} \mathrm{WO}_{4}$ precursor. T.R. Machado (T.R.M.), N.G. Macedo (N.G.M.), M. Assis (M.A.) and S. Maya-Johnson (S.M.J.) performed the TEM experiments, collected the corresponding images and videos. G.M.F. performed the MD simulations. J.A., E.L., M.S, E.Z.S, G.M.F and T.R.M. discussed the results. G.M.F. drafted the manuscript. All authors read and approved the final manuscript. 


\section{Competing interests}

The authors declare no competing interests.

\section{Additional information}

Reprints and permissions information is available at www.nature.com/reprints.

Correspondence and requests for materials should be addressed to E. Z. da Silva at zacarias@ifi.unicamp.br

Supplementary information is available for this paper at (disclose DOI address for article) and includes:

- S1. Experimental Methodology: Description of conditions for the preparation of Ag NPs as well as to promote their sintering by EB of a TEM. Video illustrating the sintering process of Ag NPs created by in situ time-resolved TEM images.

- S2. MD simulations: Technical details of MD simulations.

- S3. Sintering of Simulated Ag NPs: Additional analysis of simulated Ag NPs. 


\section{Supporting information for:}

\section{Connecting Theory with Experiment to Understand the Sintering Processes of $\mathbf{A g}$ Nanoparticles}

Edison Z. da Silva, ${ }^{*, \dagger}$ Giovani M. Faccin, ${ }^{\ddagger}$ Thales R. Machado, Marcelo Assis, Nadia G. Macedo, Santiago Maya-Johnson, "Júlio C. Sczancoski, "Juan Andrés,,$^{\S}$ Elson Longo, $₫$ and Miguel A. San-Miguel|

†Institute of Physics "Gleb Wataghin", UNICAMP, CP 6165, 13083-9859, Campinas - SP,

$$
\text { Brazil }
$$

$\ddagger$ Faculdade de Ciências Exatas e Tecnológicas, Universidade Federal da Grande Dourados Unidade II, CP 533, 79804-970, Dourados - MS, Brazil

\CDMF, LIEC, Federal University of São Carlos (UFSCar), P.O. Box 676, 13565-905 São Carlos, SP, Brazil

$\S$ Department of Analytical and Physical Chemistry, University Jaume I (UJI), Castelló 12071, Spain

||Instituto de Química, UNICAMP, CP 6165, 13083-970, Campinas - SP, Brazil E-mail: zacarias@ifi.unicamp.br 


\section{S1. Experimental Methodology}

The initial precursor comprising of $\alpha-\mathrm{Ag}_{2} \mathrm{WO}_{4}$ nanorods were synthesized by chemical precipitation in DMSO in accordance with the methodology described by Sczancoski et al. ${ }^{\text {S1 }}$ For the in situ TEM experiments, the resulting $\alpha-\mathrm{Ag}_{2} \mathrm{WO}_{4}$ powder was redispersed in water by sonicating for $5 \mathrm{~min}$, and then one drop of the dispersion was applied to a 300-mesh Cu grid coated with an ultrathin carbon support. The TEM experiments was carried out using a TITAN Themi Cubed double-corrected microscope (ThermoFisher, Scientific, USA), equipped with a gun Monochromater and X-FEG high brightness emitter gun operating at $300 \mathrm{kV}$. The microscope is located in the facilities of the Brazilian Nanotechnology National Laboratory (LNNano) in the National Center of Research in Energy and Materials (CNPEM). For the growth of mother $\mathrm{Ag}$ particles, the entire $\alpha-\mathrm{Ag}_{2} \mathrm{WO}_{4}$ nanorod was illuminated for a few minutes by the electron beam generated during TEM operation. This step causes the extrusion and reduction of $\mathrm{Ag}$ species from the crystalline lattice of the precursor oxide and the subsequent crystallization of $\mathrm{Ag}$ particles at the surface of the $\alpha-\mathrm{Ag}_{2} \mathrm{WO}_{4}$ nanorods. $\mathrm{A}$ gradually converging electron beam was used to illuminate the mother Ag particles necessary to promote an explosive reaction where many Ag NPs are fragmented. These NPs are then caught by the carbon film of TEM grid. A continuous irradiation of these segregated NPs for long exposure time ( $0 \mathrm{~s}$ to $253 \mathrm{~s})$ was used to promote the dynamic bonding events. The The High Resolution Transmission Electron Microscopy (HRTEM) experiments were carried out using Spot size 4 and a Dose rate of approximately $6 \times 10^{5} \mathrm{~A} / \mathrm{m}^{2}\left(\approx 3.6 \times 10^{6} \mathrm{e} / \mathrm{nm}^{2} \mathrm{~s}\right)$.

\section{S2. MD simulations}

Two Ag NP models with the same characteristics as the ones measured in the experiments were constructed; Fig. S1 shows two views of the models, illustrating the faceting. After being produced, the simulated Ag NPs were annealed to $500 \mathrm{~K}$ along $0.5 \mathrm{~ns}$ and then relaxed at this temperature for another $0.5 \mathrm{~ns}$, after which they were used for the temperature study 
illustrated in Figs. S2 and S3.

The simulated Ag NPs were constructed as closely as one can infer from the 2D experimental images. Their dimensions are: NP1: 3,8 x 3,7 x $4.3 \mathrm{~nm}$ and NP2: 4,1 x $4.1 \times 5.5 \mathrm{~nm}$. Both show (111) and (220) surface planes, whose areas were shaped after Wulff construction criteria.

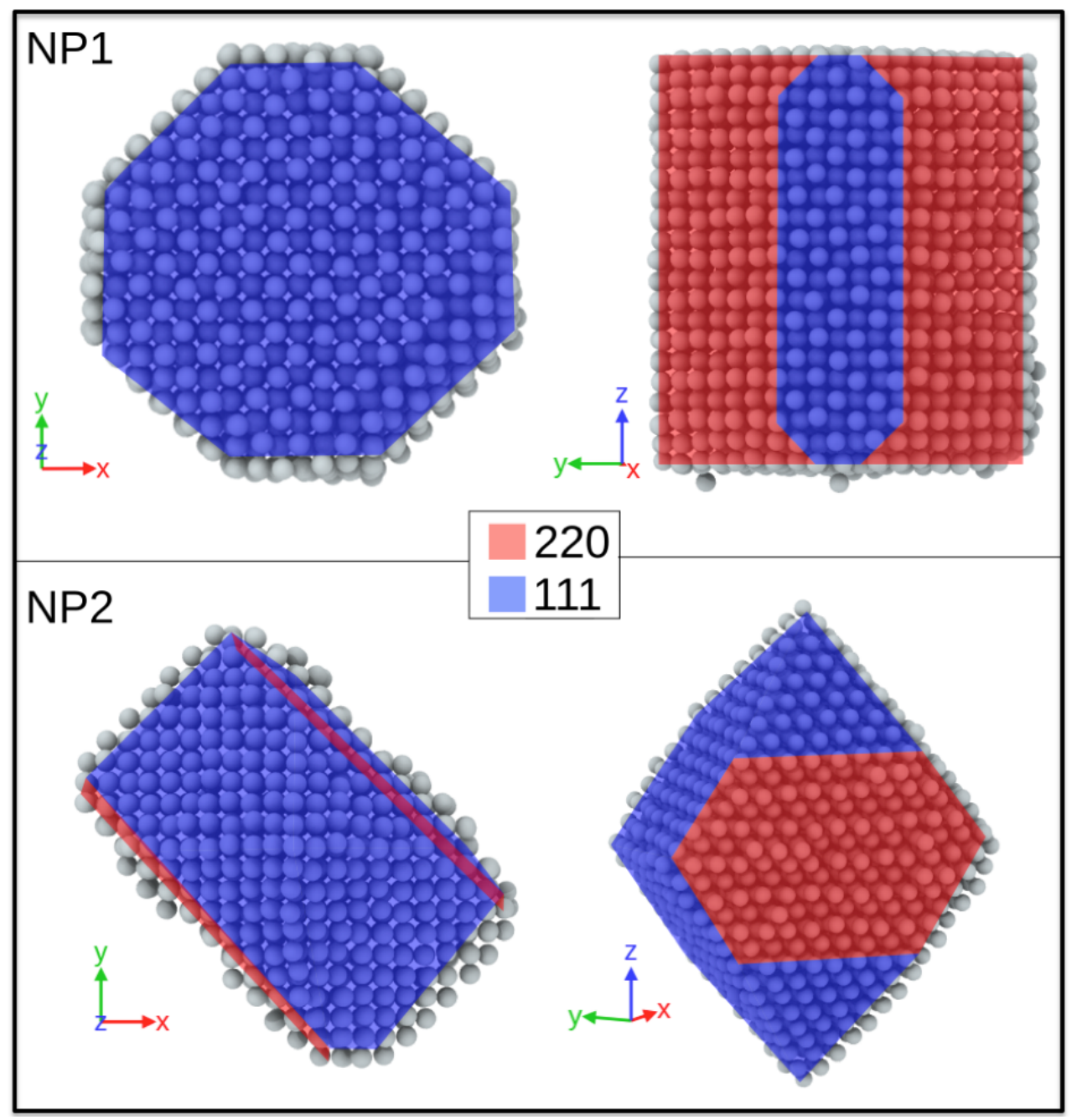

Figure S1: Models for the Ag NPs at $500 \mathrm{~K}$ showing the exposed crystalline planes.

In order to understand the behavior of surface atoms, a MD temperature study was performed for each isolated Ag NP model. The results are shown in Fig. S2 for NP1 and Fig. S3 for NP2. This study shows that, for both NPs, as expected, atoms at the (220) facets melt first, therefore they are more mobile at any temperature, which is very important 
in the sintering process. For isolated NPs, the important effect is the surface melting. Fig. S4 shows the experimental image of the Ag NPs (left) before sintering, clearly indicating blurred (220) surfaces and sharp (111) surfaces, along with the simulated images (right) that map the mobility of (220) atoms at (220) surface planes, indicating a pre-melting of (220) surfaces before the (111) ones.

For the sintering simulations the structures were extracted from the study of isolated models at $700 \mathrm{~K}$ and released close to each other. The temperature was kept constant at 700 $\mathrm{K}$ and the equations of motion were integrated for up to $290 \mathrm{~ns}$. The computer experiment was done using canonical ensemble molecular dynamics through Nosé-Hoover thermostat chains. ${ }^{\text {S2-S4 }}$ Visualizations were created using the Ovito package, ${ }^{\mathrm{S} 5}$ including the common neighbor analysis (CNA) of Dana et. al. ${ }^{\text {S5 }}$ The timestep adopted for the integration of the equations of motion was $1 \mathrm{fs}$, which is small enough to avoid significant errors due to the numerical integration process. The MD simulations were performed using the LAMMPS package $^{\mathrm{S6-S9}}$ and the embedded atom model (EAM), ${ }^{\mathrm{S} 9-\mathrm{S} 13}$ with the parametrization for $\mathrm{Ag}$ from Sheng et al., ${ }^{\text {S14 }}$ which was recently used to study Ag NPs under similar conditions. ${ }^{\text {S15-S18 }}$

\section{S3. Sintering of Simulated Ag NPs}

The simulated Ag NPs at $700 \mathrm{~K}$ were put close and oriented so that their attachment would occur in a setup similar to that measured in the experiment. Sintering started immediately and was simulated for a total time of 290 ns. The two particles touched with an angle of $40^{\circ}$, the exposed (220) surface of NP1 touching with the sharp tips of the corner formed by the (111) planes of NP2. Figure S5 shows the structures just before touching.

Simulations were performed for a long time interval $\Delta t=290 \mathrm{~ns}$. The total simulation presented many interesting features that are interesting, novel and help the understanding of experimental results. There are three different time intervals were interesting features emerged. 


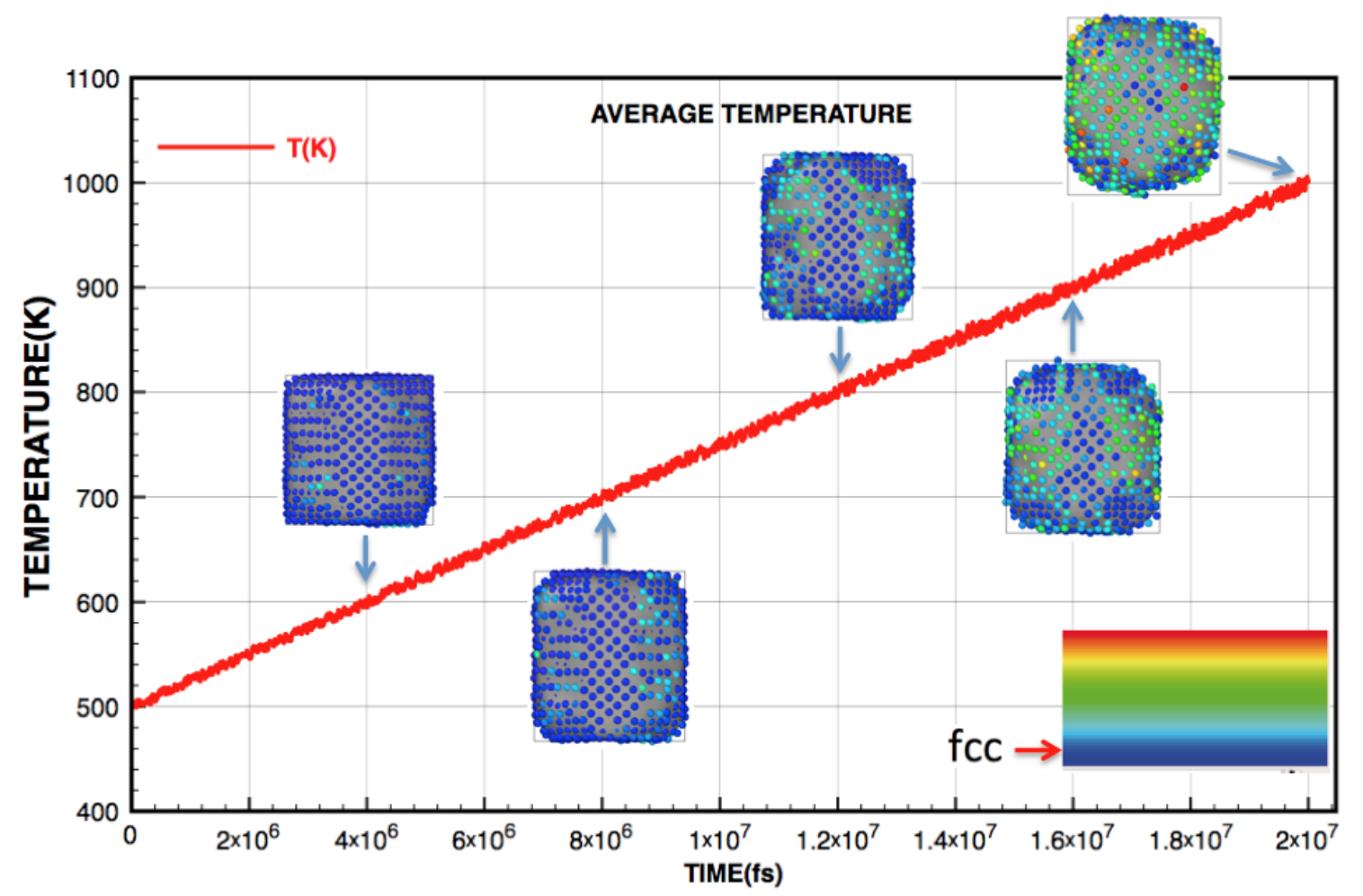

Figure S2: Temperature study of NP1, from $500 \mathrm{~K}$ to $1100 \mathrm{~K}$.

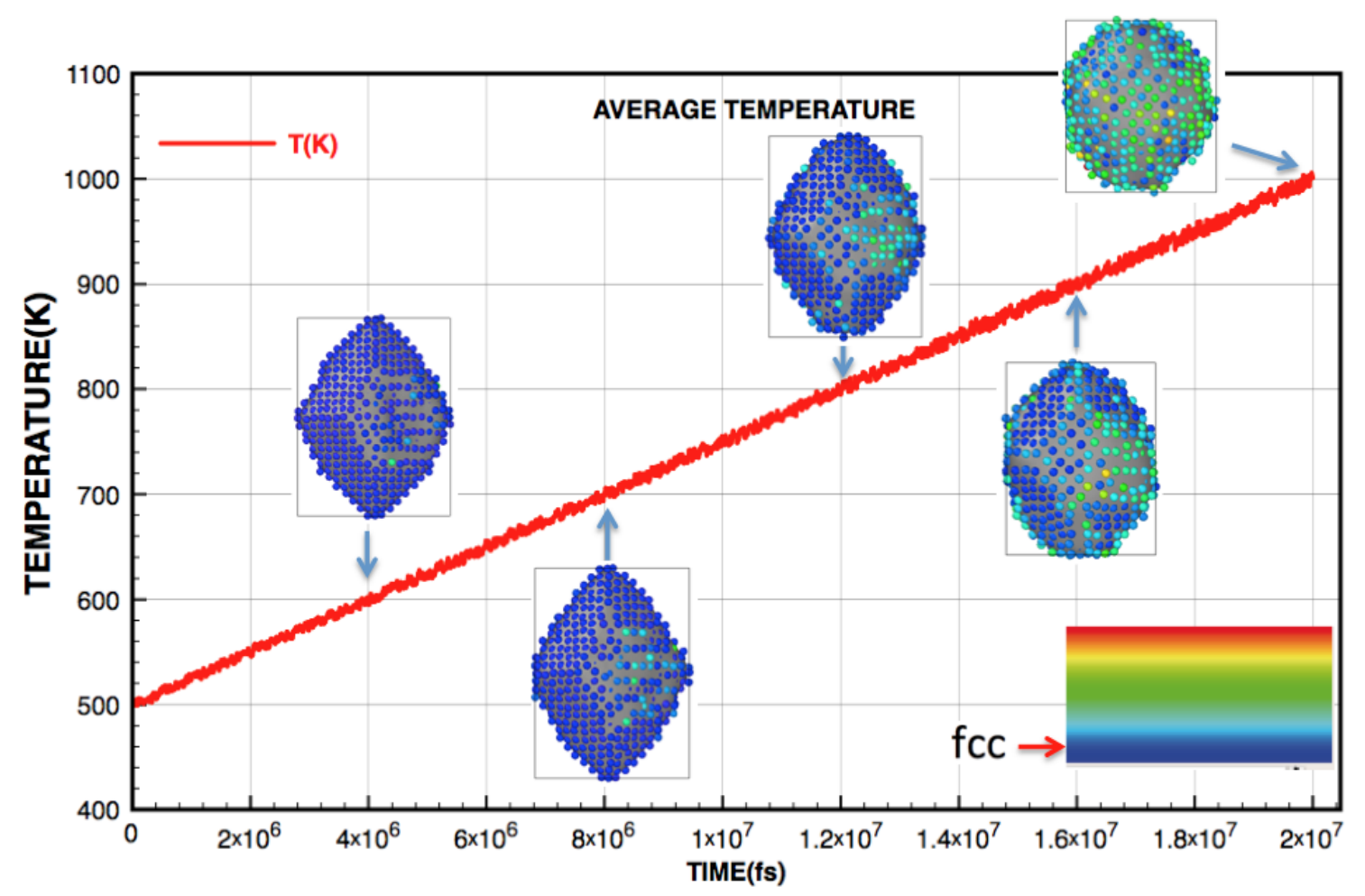

Figure S3: Temperature study of NP2, from $500 \mathrm{~K}$ to $1100 \mathrm{~K}$. 


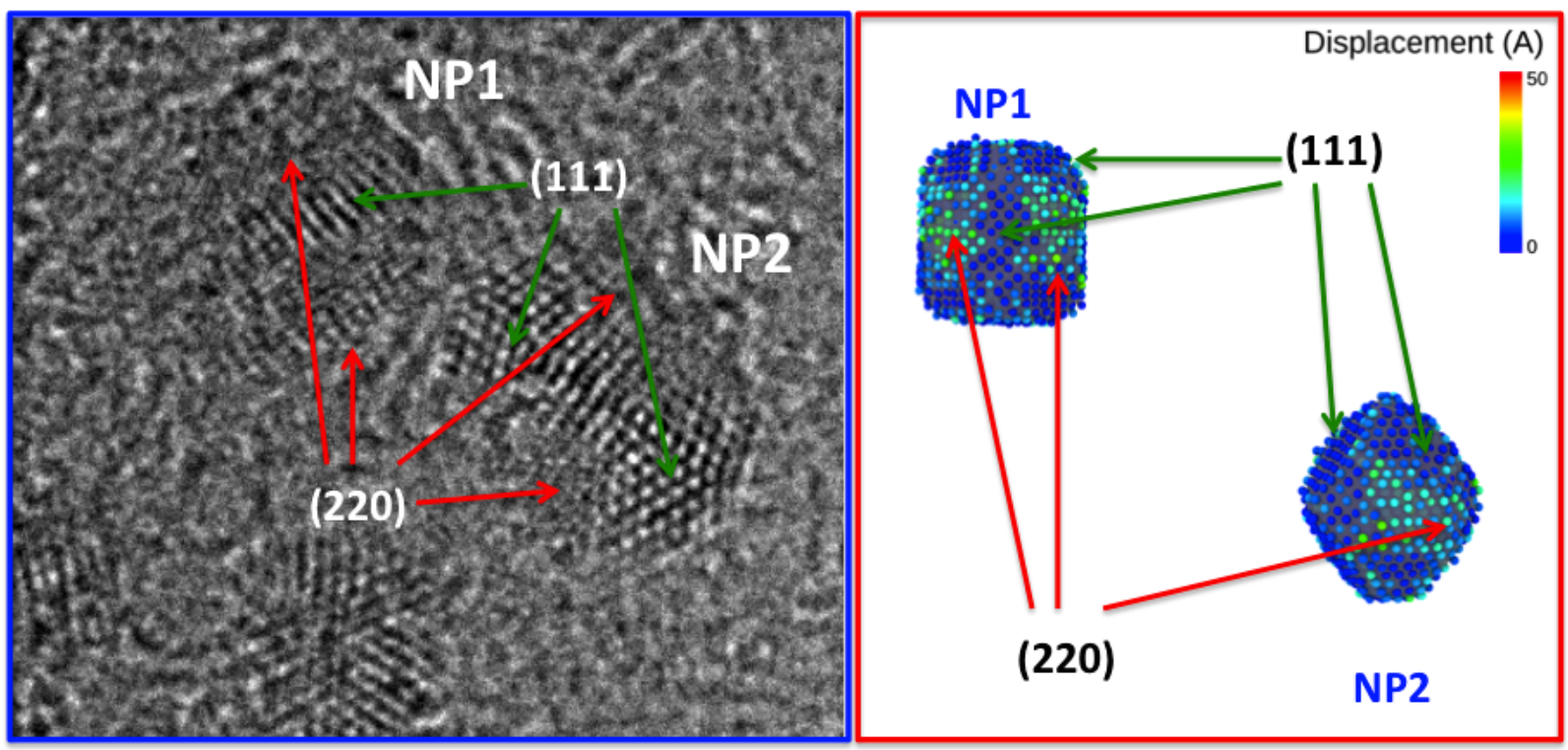

Figure S4: NP1 and NP2 in the experiment (left, blue box) and the simulated Ag NPs (right, red box), color coded showing the mobility of atoms at the (220) facets.
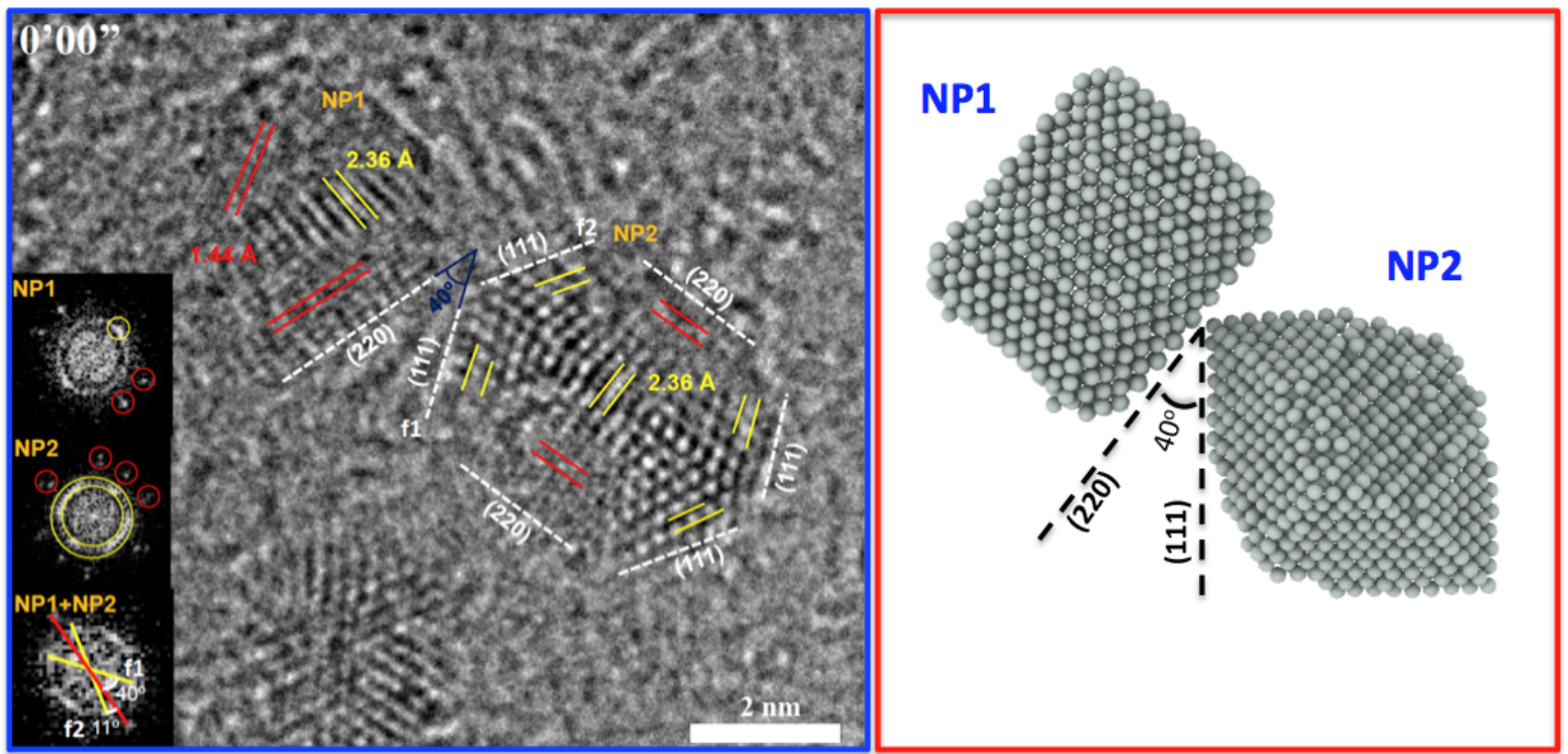

Figure S5: Nanoparticles NP1 and NP2 in the experiment (left, blue box). Simulated Ag NPs (right, red box), prior to the start of coalescence process. 


\section{Short time interval: $\Delta t=5 \mathrm{~ns}$.}

A detailed evolution, at short times, just after the beginning of the attachment process of the Ag NPs is displayed in Fig. S6. The first observation, at time of $\Delta t=0.2 \mathrm{~ns}$ is a most striking feature, the rotation of NP2 with respect to NP1. The (111) surface planes that are rather stable just rotate. The interface shows a not ordered region were facets merge and the atoms that are not in (111) faces tend to evolve to enlarge the neck.

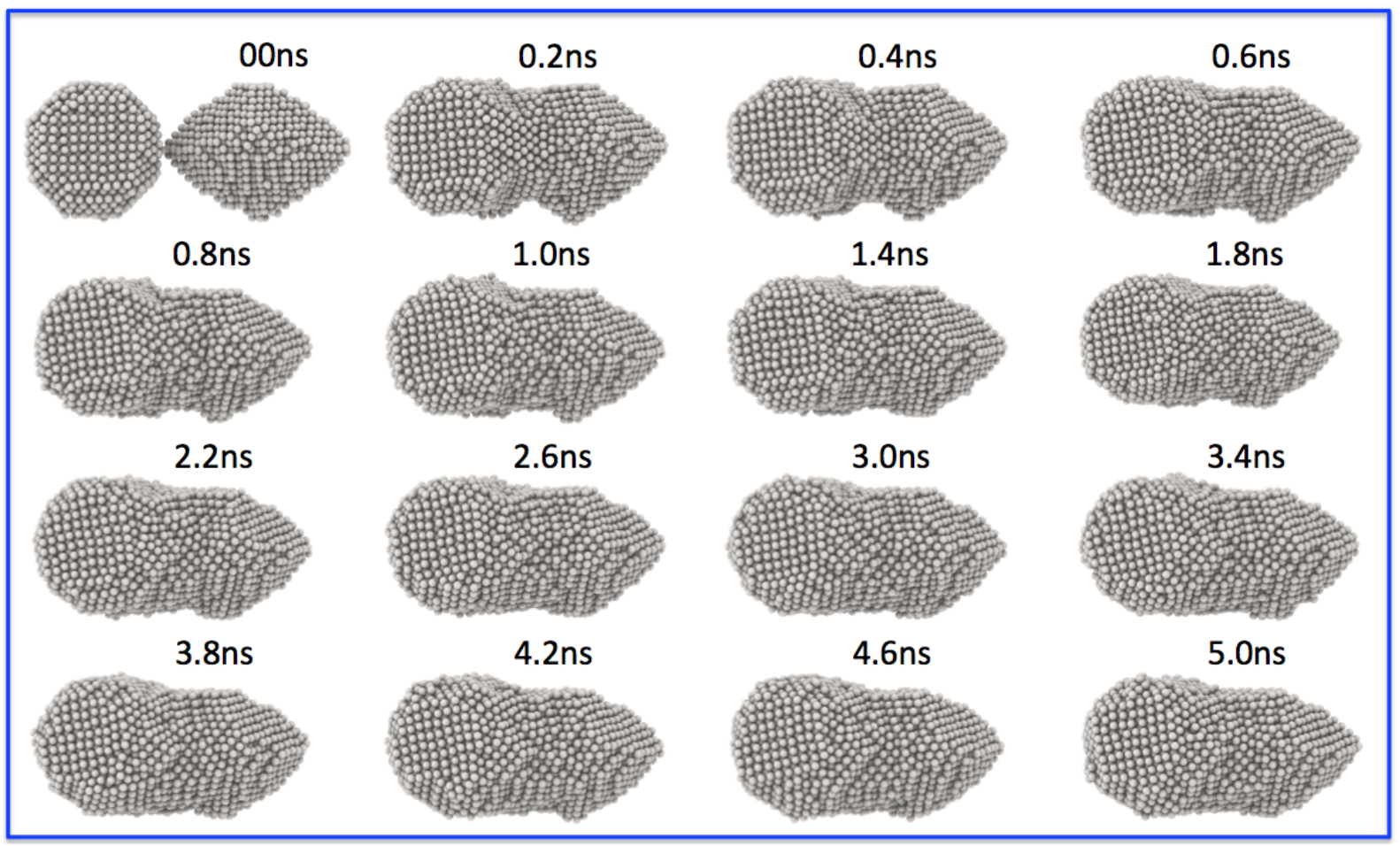

Figure S6: Short time evolution $(\Delta t=5 \mathrm{~ns})$ of the attachment process of NP1 and NP2. Snapshots are labeled by their occurrence time.

The rotation just discussed can be clearly seen in Fig. S7 were the initial structures of the two NPs are displayed in two views ( $x y$ plane in the blue box and $y z$ plane in the red box) and the first two subsequent snapshots $(t=0.2 \mathrm{~ns}$ and $t=0.4 \mathrm{~ns})$ clearly show the sudden rotation, indicated by the red arrow and that it continues to evolve as $t=5$ ns image shows.

The simulations provide a valuable tool namely the CNA analysis that can be performed 


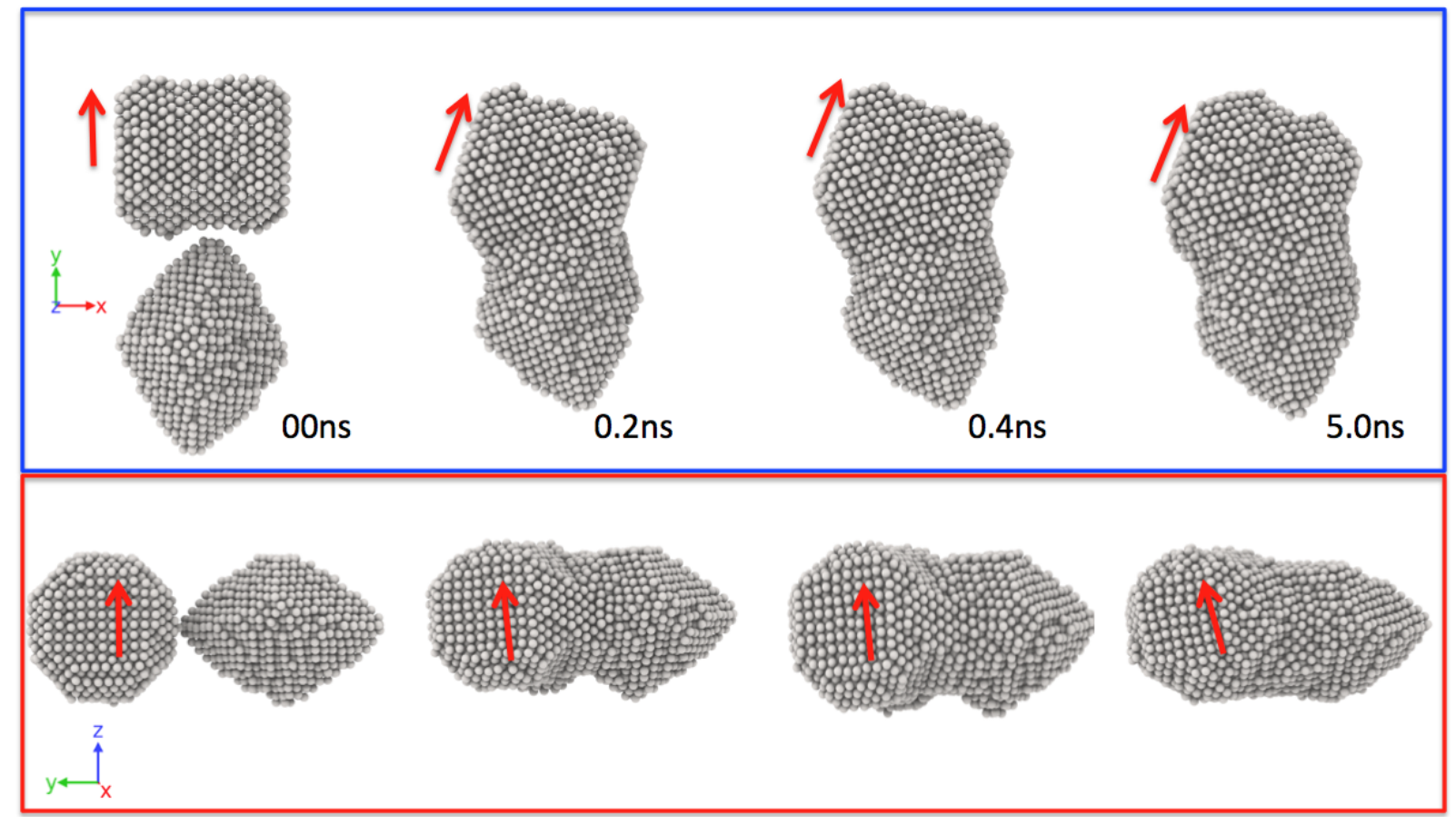

Figure S7: Details of rotation of NP2. Top, blue box shows the $x y$ plane view. Bottom, red box shows the $-y z$ plane view.

for plane cuts of the structure. The same sequence of Fig. S6 is shown for a planar cut of the former structures. Fig. S8 shows how the internal structure and in particular the interface evolves. The most striking and interesting feature starts with the formation of a disordered interface at $\Delta t=0.2 \mathrm{~ns}$. Besides this disordered interface, the coalescence process promotes the formation of other disordered regions as shown at $t=0.4 \mathrm{~ns}$, this defected formation evolves to formation of a hcp stacking fault in the region of the previous NP1. Stacking faults tend to be stable defects and difficult to heal back into fcc ordered structures, on the other hand they tend to propagate through out the NP.

\section{Intermediate time interval: $\Delta t=70 \mathrm{~ns}$.}

The disordered interface can be considered as a signature of a disorder mediated coalescence and occurred followed by the sudden rotation of NP1. Figure S9 shows at the top blue box, the evolution of the structure of the Ag NP. It clearly displays the rotation previously 


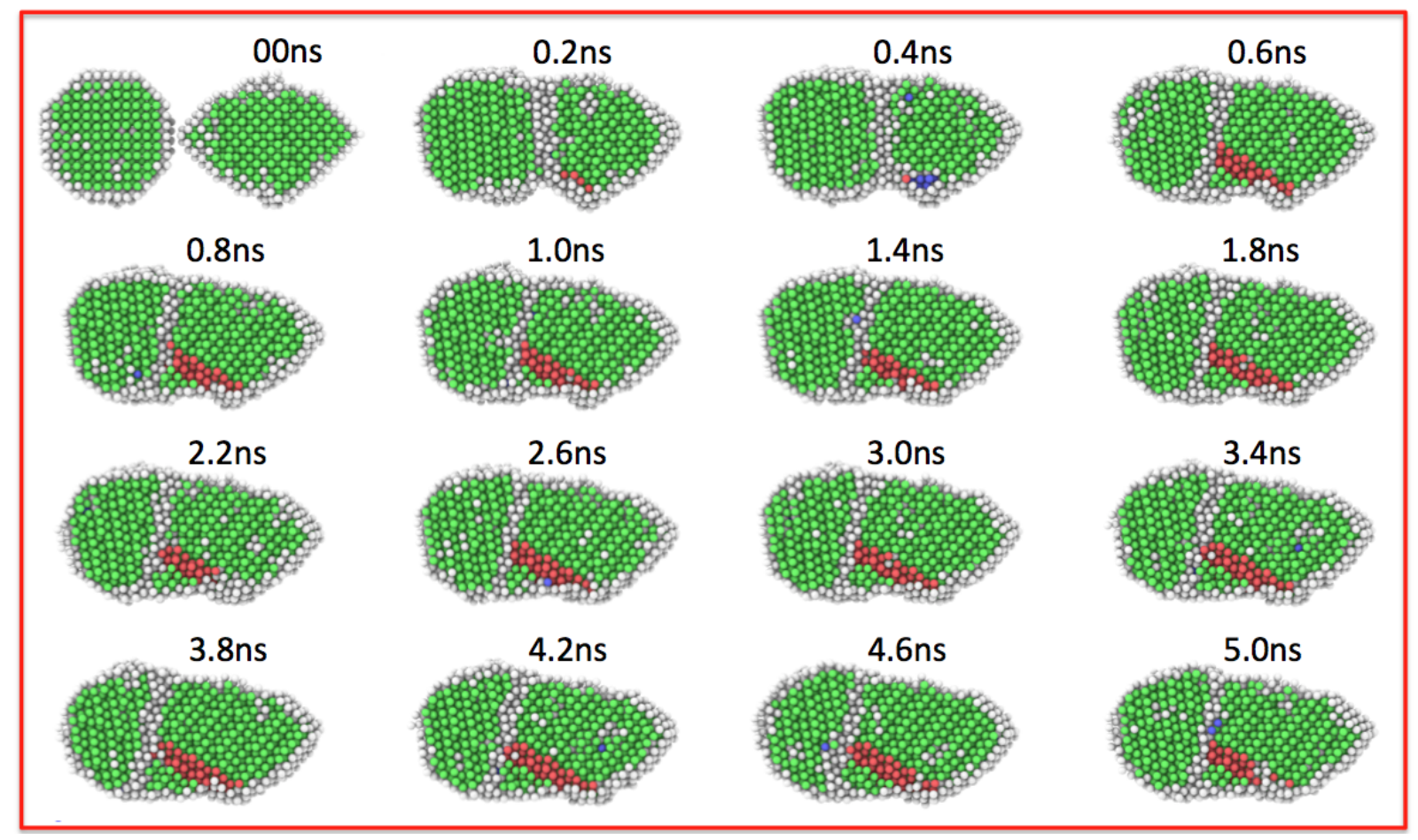

Figure S8: CNA slice cut evolution for short time interval $(\Delta t=5 \mathrm{~ns})$. It displays the evolution of the disordered interface and the formed stacking faults. Snapshots are labeled by their occurrence time. 
described and also shows the enlargement of the interface neck, caused by the surface melting and movement of the surface atoms but also and most importantly shows the evolution of the two important features of this process, namely, the disordered interface and the formed hcp stacking fault. The evolution described before in the short time behavior continued for up to $t=60 \mathrm{~ns}$, when a dramatic change in structure occurred, the healing of the disordered interface into an ordered fcc interface while the hcp stacking fault growth and propagation throughout the Ag NP extension takes place, forming two disordered surface regions that have mobile surface particles that are more free to participate in the surface atom diffusion.

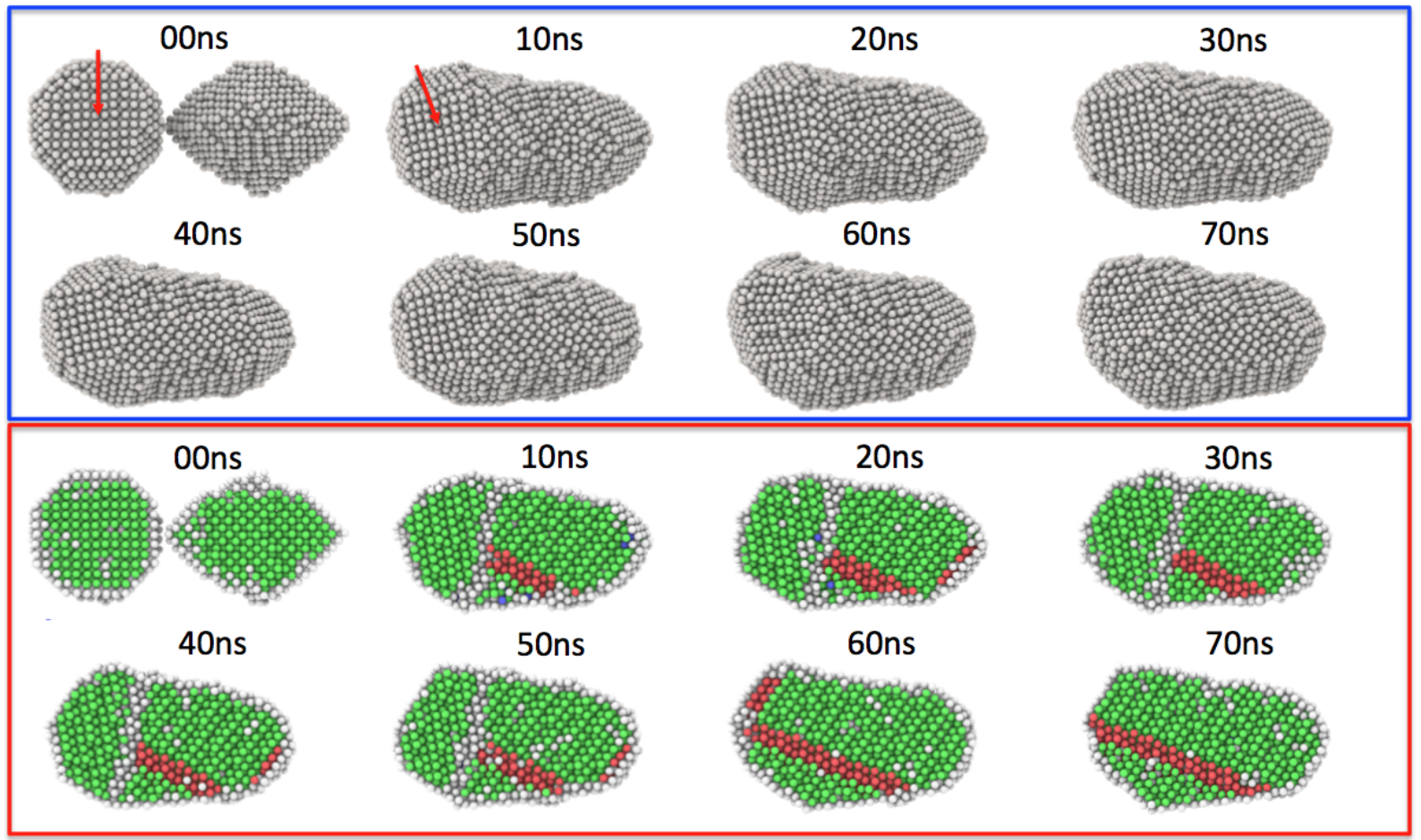

Figure S9: Structure and CNA slice view of evolution of the Ag NP for the intermediate time interval ( $\Delta t=70 \mathrm{~ns}$ ). Top blue depicts the structures and bottom frames display the CNA slice views.

The surface diffusion of atoms occurs by the movement of surface atoms from the more energetic surfaces while the more stable (111) surface atoms remain ordered. This can be seen in Fig. S10 where the bottom red box shows the atoms and their movement from the position of the previous frame. One can clearly see that the movement is large overall in 
frame at $t=10 \mathrm{~ns}$ because the NP1 is rotating while surface atoms move. After this point, movement occurs mostly at the other surfaces and interfaces with (111) ordered surface regions.

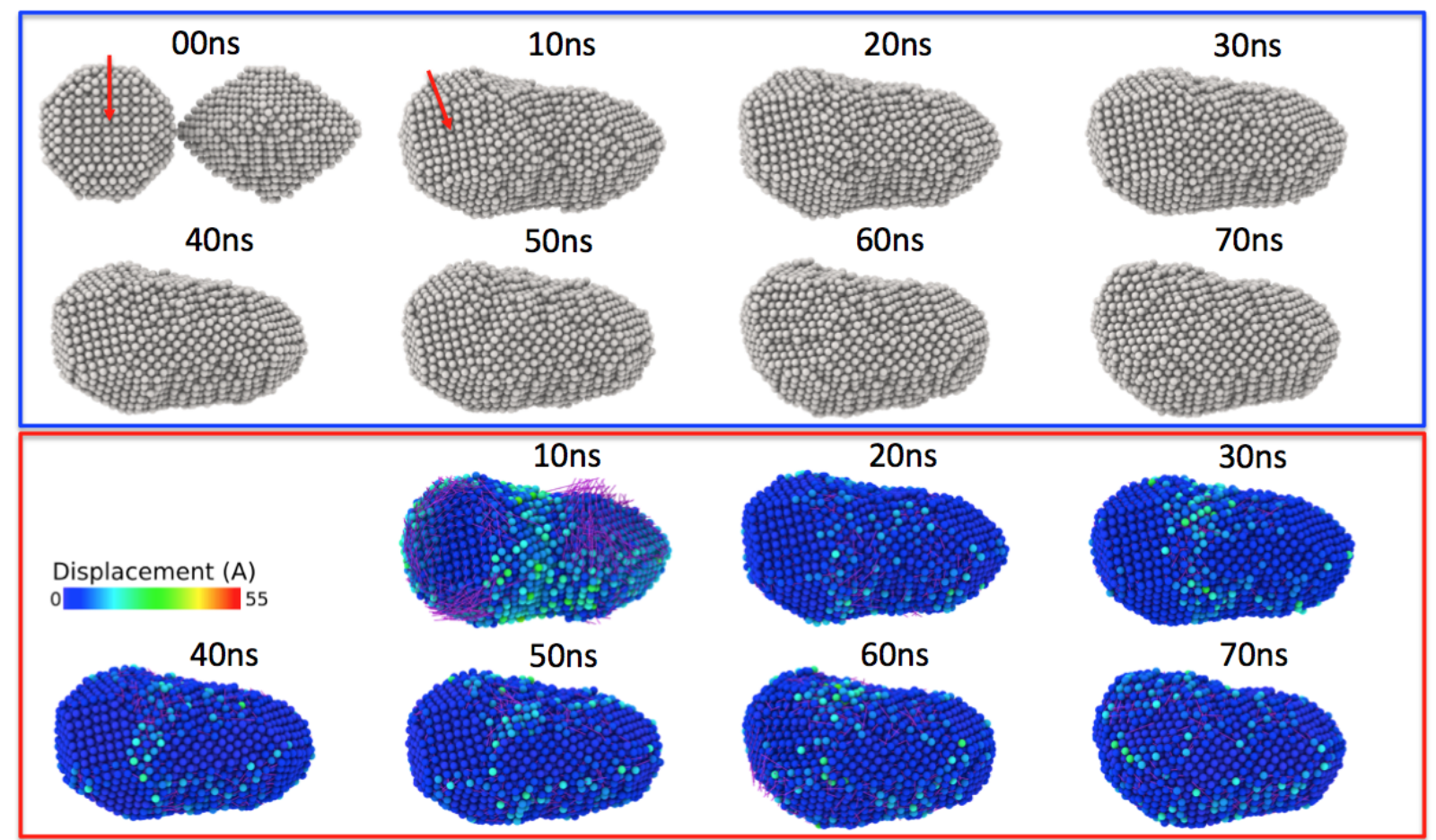

Figure S10: Structure and displacement of atoms during sintering for the intermediate time interval ( $\Delta t=70 \mathrm{~ns}$ ). Top (blue box) depicts the structures and bottom (red box) frames display the atoms and their displacement. 


\section{References}

(S1) Sczancoski, J. C.; et al., to be published.

(S2) Nosé, S. A molecular dynamics method for simulations in the canonical ensemble. Molecular Physics 1984, 52, 255-268.

(S3) Nosé, S. A unified formulation of the constant temperature molecular dynamics methods. The Journal of Chemical Physics 1984, 81, 511-519.

(S4) Hoover, W. G. Canonical dynamics: Equilibrium phase-space distributions. Phys. Rev. A 1985, 31, 1695-1697.

(S5) Honeycutt, J. D.; Andersen, H. C. Molecular dynamics study of melting and freezing of small Lennard-Jones clusters. The Journal of Physical Chemistry 1987, 91, 49504963.

(S6) Plimpton, S. Fast Parallel Algorithms for Short-Range Molecular Dynamics. Journal of Computational Physics 1995, 117, 1-19.

(S7) Brown, W. M.; Wang, P.; Plimpton, S. J.; Tharrington, A. N. Implementing molecular dynamics on hybrid high performance computers - short range forces. Computer Physics Communications 2011, 182, 898-911.

(S8) Brown, W. M.; Kohlmeyer, A.; Plimpton, S. J.; Tharrington, A. N. Implementing molecular dynamics on hybrid high performance computers - Particle-particle particlemesh. Computer Physics Communications 2012, 183, 449-459.

(S9) Brown, W. M.; Yamada, M. Implementing molecular dynamics on hybrid high performance computers-Three-body potentials. Computer Physics Communications 2013, $184,2785-2793$.

(S10) Daw, M. S.; Baskes, M. I. Embedded-atom method: Derivation and application to impurities, surfaces, and other defects in metals. Phys. Rev. B 1984, 29, 6443-6453. 
(S11) Foiles, S. M.; Baskes, M. I.; Daw, M. S. Embedded-atom-method functions for the fcc metals $\mathrm{Cu}, \mathrm{Ag}, \mathrm{Au}, \mathrm{Ni}, \mathrm{Pd}, \mathrm{Pt}$, and their alloys. Phys. Rev. B 1986, 33, 7983-7991.

(S12) Johnson, R. A. Alloy models with the embedded-atom method. Phys. Rev. B 1989, 39, 12554-12559.

(S13) Mishin, Y.; Farkas, D.; Mehl, M. J.; Papaconstantopoulos, D. A. Interatomic potentials for monoatomic metals from experimental data and ab initio calculations. Phys. Rev. B 1999, 59, 3393-3407.

(S14) Sheng, H. W.; Kramer, M. J.; Cadien, A.; Fujita, T.; Chen, M. W. Highly optimized embedded-atom-method potentials for fourteen fcc metals. Phys. Rev. B 2011, 83, 134118.

(S15) Sun, J.; He, L.; Lo, Y.-C.; Xu, T.; Bi, H.; Sun, L.; Zhang, Z.; Mao, S. X.; Li, J. Liquid-like pseudoelasticity of sub-10-nm crystalline silver particles. Nature Materials 2014, 13, 1007-1012.

(S16) Filleter, T.; Ryu, S.; Kang, K.; Yin, J.; Bernal, R. A.; Sohn, K.; Li, S.; Huang, J.; Cai, W.; Espinosa, H. D. Nucleation-Controlled Distributed Plasticity in Pentatwinned Silver Nanowires. Small 2012, 8, 2986-2993.

(S17) Sun, G.; Xu, J.; Harrowell, P. The mechanism of the ultrafast crystal growth of pure metals from their melts. Nature Materials 2018, 1476-4660.

(S18) Huang, W. J.; Sun, R.; Tao, J.; Menard, L. D.; Nuzzo, R. G.; Zuo, J. M. Coordinationdependent surface atomic contraction in nanocrystals revealed by coherent diffraction. Nature Materials 2008, 7, 308. 